\title{
Assessing the impact of non-pharmaceutical interventions (NPI) on the dynam- ics of COVID-19: A mathematical modelling study in the case of Ethiopia
}

\author{
Bedilu Alamirie Ejigy 1 , Manalebish Debalike $\mathrm{Asfaw}^{2}$, Lisa Cavalerie $^{3,4}$, Tilahun Abebaw ${ }^{2}$, Mark Nanyingi ${ }^{3,5}$, \\ Matthew Baylis ${ }^{3}$ \\ ${ }^{1}$ Department of Statistics, College of Natural and Computational Sciences, Addis Ababa University, Ethiopia \\ 2 Department of Mathematics, College of Natural and Computational Sciences, Addis Ababa University, Ethiopia \\ ${ }^{3}$ Department of Livestock and One Health, Institute of Infection, Veterinary and Ecological Sciences, University of \\ Liverpool, Liverpool, United Kingdom \\ ${ }^{4}$ International Livestock Research Institute, Addis Ababa, Ethiopia \\ ${ }^{5}$ Department of Epidemiology and Public Health, School of Public Health, University of Nairobi, Kenya
}

\begin{abstract}
The World Health Organisation (WHO) declared COVID-19 a pandemic on March 11, 2020 and by November 14, 2020 there were 53.3M confirmed cases and 1.3M reported deaths in the world. In the same period, Ethiopia reported $102 \mathrm{~K}$ cases and $1.5 \mathrm{~K}$ deaths. Effective public health preparedness and response to COVID-19 requires timely projections of the time and size of the peak of the outbreak. Currently, in the absence of vaccine or effective treatment, the implementation of NPIs (non-pharmaceutical interventions), like hand washing, wearing face coverings or social distancing, is recommended by WHO to bring the pandemic under control. This study proposes a modified Susceptible Exposed Infected and Recovered (SEIR) model to predict the number of COVID-19 cases at different stages of the disease under the implementation of NPIs with different adherence levels in both urban and rural settings of Ethiopia. To estimate the number of cases and their peak time, 30 different scenarios were simulated. The results reveal that the peak time of the pandemic is different in urban and rural populations of Ethiopia. In the urban population, under moderate implementation of three NPIs the pandemic will be expected to reach its peak in December, 2020 with 147,972 cases, of which 18,100 are symptomatic and 957 will require admission to an Intensive Care Unit (ICU). Among the implemented NPIs, increasing the coverage of wearing masks by $10 \%$ could reduce the number of new cases on average by one-fifth in urban-populations. Varying the coverage of wearing masks in rural populations minimally reduces the number of cases. In conclusion, the projection result reveals that the projected number of hospital cases is higher than the Ethiopian health system capacity during the peak time. To contain symptomatic and ICU cases within health system capacity, the government should give attention to the strict implementation of the existing NPIs or impose additional public health measures.
\end{abstract}

\footnotetext{
${ }^{1}$ Corresponding Author: bedilu.alamirie@aau.edu.et
} 


\section{Introduction}

The novel Severe Acute Respiratory Syndrome Coronavirus 2 (SARS-CoV-2) was first discovered in Wuhan, China in December 2019 WHO (2020). The associated disease, COVID-19 was declared as a pandemic by the World Health Organisation (WHO) on March 11, 2020. It has led to over 53.5 million confirmed cases and 1.3 million deaths across the globe by November 14, 2020 WHO (2020). In Africa, the burden and impacts of the pandemic are less compared with Europe and the USA. This may be attributed to late arrival of the pandemic, low seeding rate, youthful demographics and possible prior immunity to coronavirus-like infections Njenga et al. (2020). Nevertheless, the pandemic is accelerating in Africa and passed 2.0 million confirmed cases on November 13, 2020 according to the Africa-CDC (2020) situation report. In Ethiopia, the first imported COVID-19 case, a Japanese person who travelled from Burkina Faso, was detected and reported by the Ministry of Health on March 13, 2020, Institute (2020). To control the spread of the disease, the Ethiopian government took immediate action, by closing schools, banning sporting events and mass gatherings, three days later. It took 79 days to reach the first 1000 cases, but 10,000, 20,000, 40,000 and 80,000 cases were attained in shorter periods of 51, 16, 18 and 44 days, respectively. This shows that the number of cases has been increasing over time, surpassing 100,000 by the mid of November, 2020. As the pandemic accelerates globally, many African countries, including Ethiopia, have been implementing different non-pharmaceutical interventions (NPIs) to contain the spread of the disease. The WHO is leading a global COVAX initiative for accelerated development of promising vaccine candidates that may be available in early 2021 , and experimental therapies are also undergoing clinical trials for safety before licensing. However, at this point in time, the most effective means to control the spread of COVID-19 remains the implementation of different NPIs to break chains of transmission Fong et al. (2020b); Ryu et al. (2020); Lai et al. (2020); Flaxman et al. (2020); Ferguson et al. (2020). The transmission pathways of COVID-19 from person to person are: i) close contact through respiratory droplet, ii) direct contact with infected persons, and iii) contact with contaminated formites (objects and surfaces) Kassa et al. (2020). Public health measures are intended to diminish these transmission mechanisms.

Mathematical models have been previously used with success in understanding the transmission dynamics and control mechanisms of infectious diseases (Anderson and May (1979)). To understand the early transmission dynamics of COVID-19 under different scenarios, a number of mathematical models have been proposed in the literature, Prem et al. (2020); Li et al. (2020); Walker et al. (2020); Ivorra et al. (2020); Ngonghala et al. (2020); Nicholas et al. (2020). Those epidemiological and mathematical models contributed important insight for public health decisionmakers to enforce different mitigation strategies in different countries. As COVID-19 has spread worldwide, most countries are utilising mathematical models to inform on public health measures. Many countries (for example the UK, China, Germany, USA, Morocco) revised their public health measures based on COVID-19 modelling results. We believe that, due to the difference in the age-structure of the population, social interaction and life style in Ethiopia, mathematical models developed in other countries may not work to study the dynamics of disease in lower income settings. This study employs the use of mathematical models to simulate the spread and interruption of transmission of the disease in Ethiopia.

Assessment of implemented and proposed intervention strategies for combating COVID-19, and predicting the number of new cases and deaths is a major challenge to both the public and scientific community. Banholzer et al. 
(2020) and Flaxman et al. (2020) estimated the impact of NPIs based on confirmed cases of COVID-19 in several countries. Similarly, Ferguson et al. (2020) studied the impact of NPIs to reduce COVID-19 mortality and healthcare demand. To our knowledge, there has been very limited study of the impact of NPIs on the disease dynamics in the local context of Ethiopia. The only published study, done by Siraj et al. (2020), focused on small, medium and large clusters of the population with social distancing face masking and contact tracing implementation at different proportion. This study did not quantify the impact of hand washing measures on transmission dynamics. Further, their study did not provide the estimated number of cases under different stages of the disease. Takele (2020) implements the Autoregressive Integrated Moving Average (ARIMA) modeling to project COVID-19 prevalence patterns in East Africa Countries, mainly Ethiopia, Djibouti, Sudan and Somalia. While, her projection model did not take into account the impact of NPIs with different adherence level on the predicted number of cases.

Our study proposes a modified mathematical model of the classic SEIR model (Anderson and May (1979)) and this modified model is used to compare the effect of different NPIs individually and in combination. The proposed model classifies the human population into eight non-overlapping stages of infection: Susceptible $(S)$, Exposed $(E)$, Asymptomatic infectious $\left(I_{a}\right)$, Symptomatic infectious $\left(I_{s}\right)$, Isolation of cases with mild/moderate health condition (at home or hospital) $\left(H I_{m}\right)$, Hospitalized with critical health condition $\left(H_{c}\right)$, Recovered from the disease $(R)$, and Death due to COVID-19 $(D)$.

Our proposed model (1) has a number of advantages: i) provide estimated time and size of the peak under the implementation of NPIs with different adherence level in urban and rural population settings, ii) proposed model differentiates asymptomatic and symptomatic infectious which influences the number of ICU cases and death due to the disease, iii) the effect of indirect transmission of the disease through contaminated environment is taken into account, and iv) provide the estimated impact of individual and synergy public health measures on the dynamics of the disease. The projected number of people in each stage of the disease at the peak period and the time of the peak in urban and rural areas of Ethiopia helps the government to choose and enforce better intervention mechanisms.

\section{Materials and Methods}

\subsection{Representation of Proposed Mathematical Model}

In our modeling framework, the population is divided into different compartments according to the infection status of individuals: susceptible $(\mathrm{S})$, exposed $(\mathrm{E})$, asymptomatic infected $\left(I_{a}\right)$, symptomatic infected $\left(I_{s}\right)$, isolated at home or hospital with moderate health condition $\left(I H_{m}\right)$, hospitalized with severe health condition $\left(H_{c}\right)$, recovered from the disease (R), and death (D) due to the disease. Contaminated environment is also a means of transmission for COVID-19 as the virus can stay up to several days on different surfaces van Doremalen et al. (2020). To account for the impact of contaminated objects in the transmission, an additional compartment for contaminated environment is included. Paramter description with these assumed initial values are presented in Table 1 . Those values were extracted from a number of key papers and reports of the situation in Ethiopia (Institute (2020); Goshu et al. (2020); Baye (2020); Kebede et al. (2020); Siraj et al. (2020) ) and epidemiological scientific facts of COVID-19.

By assuming that the total population is $N=S+E+I_{a}+I_{s}+I H_{m}+H_{C}+R+D$, the corresponding system 


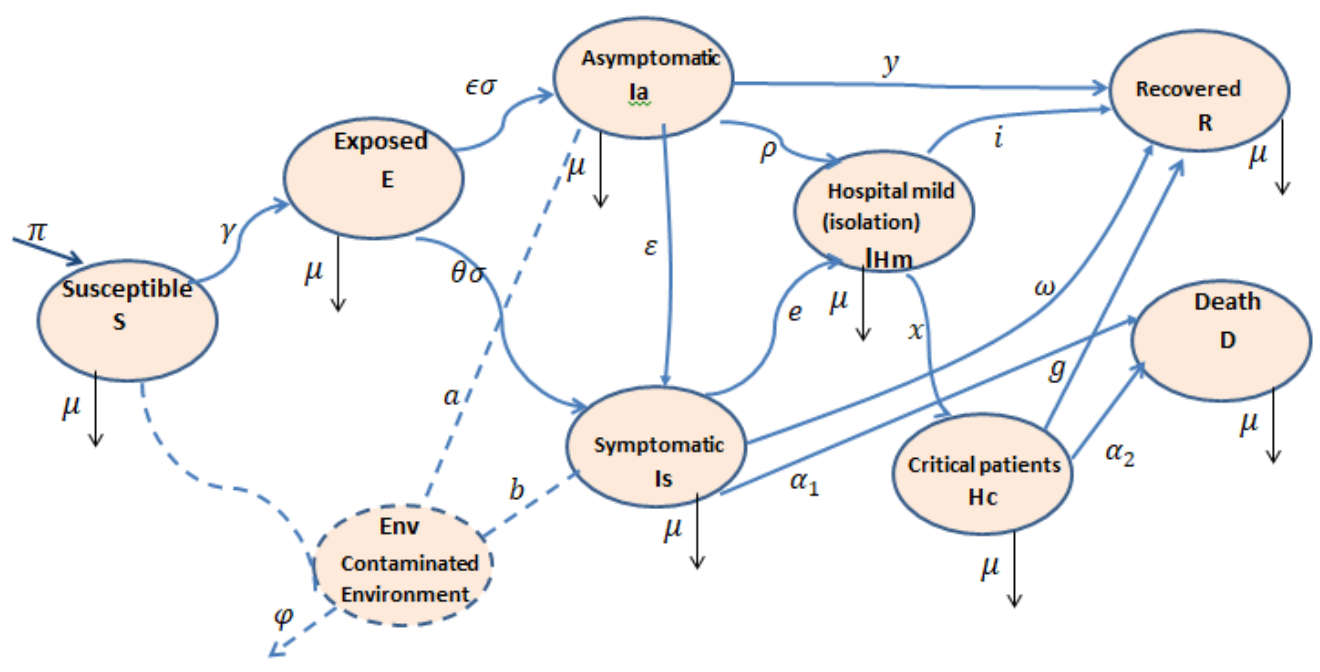

Figure 1: Flow diagram of the mathematical model showing the transition of individuals in different compartments based on infectious status

of differential equations of our proposed model is given by

$$
\begin{aligned}
\frac{d S}{d t} & =\pi-\gamma S-\mu S \\
\frac{d E}{d t} & =\gamma S-\sigma E-\mu E \\
\frac{d I_{a}}{d t} & =(1-\theta) \sigma E-\rho I_{a}-\varepsilon I_{a}-y I_{a}-\mu I_{a} \\
\frac{d I_{s}}{d t} & =\theta \sigma E+\varepsilon I_{a}-e I_{s}-\omega I_{s}-\alpha_{1} I_{s}-\mu I_{s} \\
\frac{d I H_{m}}{d t} & =\rho I_{a}+e I_{s}-x I H_{m}-i I H_{m}-\mu I H_{m} \\
\frac{d H_{c}}{d t} & =x I H_{m}-g H_{c}-\alpha_{2} H_{c}-\mu H_{c} \\
\frac{d R}{d t} & =g H_{c}+i I H_{m}+y I_{a}+\omega I_{s}-\mu R \\
\frac{d D}{d t} & =\alpha_{1} I_{s}+\alpha_{2} H_{c} \\
\frac{d E_{n v}}{d t} & =a I_{a}+b I_{s}-\varphi E_{n v}
\end{aligned}
$$

where the force of infection, $\gamma$, is obtained using the following formula:

$$
\gamma=r_{4} \beta_{1}\left(\frac{\left(\eta_{a} I_{a}+I_{s}\right)}{N}\right)+r_{4} \beta_{2}\left(\frac{E_{n v}}{E_{n v}+K}\right) .
$$

In Equation (3, $\beta_{1}$ and $\beta_{2}$ are effective contact rates leading to COVID-19, $\eta_{a}$ is relative infectiousness per contact for asymptomatic patients, $r_{4}=(1-c F M) *(1-S D) *(1-H W)$, where $S D$ represents the proportion of the population who practice physical distancing, $H W$ represents the proportion of the population who implements hand washing, $F M$ is coverage of wearing masks, $c$ is efficacy of face mask wearing at reducing transmission, and $K$ is the virus concentration in the environment that yields $50 \%$ of chance for a susceptible individual to catch the viral infection 
from that source, Kassa et al. (2020). The model accounts for a distinction between non-diagnosed individuals $I_{a}$ and $I_{s}$, who can readily spread the infection because they are not in isolation, and hospitalized individuals $H I_{m}$ and $H_{c}$, who transmit the disease less thanks to isolation and complying with strict rules, either in hospital or at home.

\subsection{Well-posedness of the System and sensitivity analysis}

To check the mathematical and biological meaningfulness of the model, both positivity and boundedness properties of the proposed model are assessed as follows. Let the domain space be defined by

$$
\Omega=\left\{\left(S, E, I_{s}, I_{a}, H_{c}, I H_{m}, R, D, E_{n v}\right) \in \mathbb{R}_{+}^{9}\right\}
$$

Theorem 2.1. $\operatorname{int}(\Omega)=\left\{\left(S, E, I_{s}, I_{a}, H_{c}, I H_{m}, R, D, E_{n v}\right) \mid S>0, E \geq 0, I_{s} \geq 0, I_{a} \geq 0\right.$, $\left.\left.H_{c} \geq 0, I H_{m} \geq 0, R \geq 0, D \geq 0, E_{n v} \geq 0\right)\right\}$ is the positive invariant set of the system [2].

The proof of theorem 2.1 is presented in the Appendix and the theorem implies well-posedness of the system.

\section{Sensitivity analysis of model parameters}

In order to identify parameters which significantly influence the model system, sensitivity analysis was performed. The uncertainty and sensitivity analysis is done by using Partial Rank Correlation Coefficient (PRCC) analysis with $\mathrm{N}=10,000$ samples for various input parameters Wu et al. (2013); Marino et al. (2008). The partial derivative of the threshold value $R_{o}$ with respect to the input parameters were computed by varying the parameters around normal values.

\subsection{System Equilibria and Stability of the System}

\subsubsection{Disease Free Equilibrium}

Equilibrium points are those points where each equation in the system in Equation (1) is equal to zero. Equating the right hand side of each equation in Equation (1) to zero will provide disease-free and endemic equilibrium points. In this subsection, the disease-free equilibrium point is described as follows.

When there is no COVID-19 in the population, the disease-free equilibrium point is given by

$$
\mathcal{E}_{0}=\left(S, E, I_{a}, I_{s}, I H_{m}, H_{c}, R, D, E_{n v}\right)=\left(\frac{\pi}{\mu}, 0,0,0,0,0,0,0,0\right)
$$

In this study, to compute the basic reproduction number, Diekmann et al. (1990), next-generation method for the disease-free equilibrium is employed. The basic reproduction number is the average number of secondary infection cases produced in a completely susceptible population by a typical infectious individual. According to the definitions stated in Diekmann et al. (1990) and van den Driessche and Watmough (2002a), in the next-generation method, $R_{0}$ is the spectral radius of the next-generation matrix which is given by

$$
R_{0}=\rho\left(F V^{-1}\right)
$$


where $F$ is the Jacobian of the rate of appearance of new infections in the infected compartment, denoted by $f$ and $V$ is the Jacobian of $v=v^{-}-v^{+}$that represents all infection transfer interactions into $\left(v^{+}\right)$and out $\left(v^{-}\right)$of these compartments.

The infection compartments of our model are $\left(E, I_{a}, I_{s}, I H_{m}, H_{c}, E_{n v}\right)$

$$
f=\left(\begin{array}{c}
\gamma S \\
0 \\
0 \\
0 \\
0 \\
0
\end{array}\right), \quad v=\left(\begin{array}{c}
(\sigma+\mu) E \\
-(1-\theta) \sigma E+(\varepsilon+y+\mu) I_{a} \\
-\theta \sigma E-\varepsilon I_{a}+\left(e+\alpha_{1}+\omega+\mu\right) I_{s} \\
-\rho I_{a}-e I_{s}+(i+x+\mu) I H_{m} \\
-x I H_{m}+\left(g+\alpha_{2}+\mu\right) H_{c} \\
-a I_{a}-b I_{s}+\varphi E_{n v}
\end{array}\right)
$$

Computing the Jacobian of $f$ and evaluating it at the disease-free equilibrium point using the force of infection, we have,

$$
F\left(\mathcal{E}_{0}\right)=\left(\begin{array}{cccccc}
0 & k_{4} \beta_{1} \eta_{a} \frac{\mu}{\pi} & k_{4} \beta_{1} \frac{\mu}{\pi} & 0 & 0 & k_{4} \beta_{2} \frac{\varphi}{K(a+b)} \\
0 & 0 & 0 & 0 & 0 & 0 \\
0 & 0 & 0 & 0 & 0 & 0 \\
0 & 0 & 0 & 0 & 0 & 0 \\
0 & 0 & 0 & 0 & 0 & 0 \\
0 & 0 & 0 & 0 & 0 & 0
\end{array}\right)
$$

and the Jacobian of $v$ evaluated at the disease free equilibrium point using the force of infection is given by the matrix

$$
V\left(\mathcal{E}_{0}\right)=\left(\begin{array}{cccccc}
\sigma+\mu & 0 & 0 & 0 & 0 & 0 \\
-(1-\theta) \sigma & (\varepsilon+y+\mu) & 0 & 0 & 0 & 0 \\
-\theta \sigma & -\varepsilon & e+\alpha_{1}+\omega+\mu & 0 & 0 & 0 \\
0 & -\rho & -e & i+x+\mu & 0 & 0 \\
0 & 0 & 0 & -x & g+\alpha_{2}+\mu & 0 \\
0 & -a & -b & 0 & 0 & \varphi
\end{array}\right) .
$$

Finding the inverse of $V$ and computing the product $F V^{-1}$, the next generation matrix is given by

$$
F V^{-1}=\left(\begin{array}{cccccc}
A & B & C & 0 & 0 & D \\
0 & 0 & 0 & 0 & 0 & 0 \\
0 & 0 & 0 & 0 & 0 & 0 \\
0 & 0 & 0 & 0 & 0 & 0 \\
0 & 0 & 0 & 0 & 0 & 0 \\
0 & 0 & 0 & 0 & 0 & 0
\end{array}\right)
$$


where for

$$
\begin{aligned}
& k_{1}=\sigma+\mu, k_{2}=\rho+\varepsilon+y+\mu \text { and }, k_{3}=e+\alpha_{1}+\omega+\mu . \\
& A=r_{4} \beta_{1} \frac{\mu}{\pi}\left(\frac{(1-\theta) \varepsilon \sigma+\theta \sigma k_{2}}{k_{1} k_{2} k_{3}}\right)+\frac{r_{4} \beta_{1} \eta_{a} \mu(1-\theta) \sigma}{\pi k_{1} k_{2}}+\frac{r_{4} \beta_{2}}{K}\left(\frac{b \theta \sigma k_{2}+(1-\theta) \sigma\left(\varepsilon b+a k_{3}\right)}{K \varphi k_{1} k_{2} k_{3}}\right) \\
& B=\frac{r_{4} \beta_{1} \varepsilon \mu}{\pi k_{2} k_{3}}+\frac{r_{4} \beta_{1} \eta_{a} \mu}{\pi k_{2}}+\frac{r_{4} \beta_{2}}{K}\left(\frac{\varepsilon b+a k_{3}}{\varphi k_{2} k_{3}}\right) \\
& C=\frac{r_{4} \beta_{1} \mu}{\pi k_{3}}+r_{4} \beta_{2} \frac{b}{K \varphi k_{3}} \\
& D=\frac{r_{4} \beta_{2}}{K \varphi}
\end{aligned}
$$

The corresponding Spectral radius of the matrix $F V^{-1}$ is given by $R_{0}=\rho\left(F V^{-1}\right)=A$, where

$$
A=\underbrace{r_{4} \beta_{1} \frac{\mu}{\pi}\left(\frac{(1-\theta) \varepsilon \sigma+\theta \sigma k_{2}}{k_{1} k_{2} k_{3}}\right)}_{R_{0 s}}+\underbrace{\frac{r_{4} \beta_{1} \eta_{a} \mu(1-\theta) \sigma}{\pi k_{1} k_{2}}}_{R_{0 a}}+\underbrace{\frac{r_{4} \beta_{2}}{K}\left(\frac{b \theta \sigma k_{2}+\epsilon \sigma\left(\varepsilon b+a k_{3}\right)}{K \varphi k_{1} k_{2} k_{3}}\right)}_{R_{0 E n v}}
$$

which can be expressed as $A=R_{0}=R_{0 a}+R_{0 s}+R_{0 E n v}$ and the quantity $R_{0}$ is the basic reproduction number of the Model (2). The quantity $R_{0}$ is the sum of the constituent reproduction numbers associated with the number of new COVID-19 cases generated by symptomatically infectious humans $\left(R_{0 s}\right)$, asymptomatically infectious humans $\left(R_{0 a}\right)$ and contaminated environment $\left(R_{0 E_{n v}}\right)$.

Theorem 2.2. The disease-free equilibrium (DFE) of model 2 is locally asymptotically stable if $R_{0}<1$, and unstable if $R_{0}>1$.

Proof. It follows from Theorem 2 of van den Driessche and Watmough (2002b)

The implication of Theorem 2.2 is that a small introduction of COVID-19 cases will not generate a COVID-19 outbreak if the basic reproduction number $\left(R_{0}\right)$ is less than unity.

Theorem 2.3. The disease-free equilibrium of model 2 is globally asymptotically stable if $R_{0} \leq 1$.

\subsubsection{Endemic Equilibrium}

The endemic equilibrium is attained in the presence of the disease, which is denoted by:

$$
\mathcal{E}=\left(S, E, I_{a}, I_{s}, I H_{m}, H_{c}, R, D, E_{n v}\right)=\left(S^{*}, E^{*}, I_{a}^{*}, I_{s}^{*}, H_{M}^{*}, H_{C}^{*}, R^{*}, D^{*}, E_{n v}^{*}\right)
$$


and the values of $E^{*}, I_{s}^{*}, H_{m}^{*}, H_{c}^{*}, R^{*}$ and $E_{n v}^{*}$ can be computed from the equations:

$$
\begin{aligned}
& \pi-r_{4} \beta_{1}\left(\frac{\eta_{a} I_{a}+I_{s}}{N}\right) S-r_{4} \beta_{2}\left(\frac{E_{n v}}{E_{n v}+K}-\mu\right) S=0 \\
& r_{4} \beta_{1}\left(\frac{\eta_{a} I_{a}+I_{s}}{N}\right) S+r_{4} \beta_{2}\left(\frac{E_{n v}}{E_{n v}+K}\right) S-\sigma E-\mu E=0 \\
& (1-\theta) \sigma E-\rho I_{a}-\varepsilon I_{a}-y I_{a}-\mu I_{a}=0 \\
& \theta \sigma E+\varepsilon I_{a}-e I_{s}-\omega I_{s}-\alpha_{1} I_{s}-\mu I_{s}=0 \\
& \rho I_{a}+e I_{s}-x I H_{m}-i I H_{m}-\mu I H_{m}=0 \\
& x I H_{m}-g H_{c}-\alpha_{2} H_{c}-\mu H_{c}=0 \\
& g H_{c}+i I H_{m}+y I_{a}+\omega I_{s}-\mu R=0 \\
& \alpha_{1} I_{s}+\alpha_{2} H_{c}=0 \\
& a I_{a}+b I_{s}-\varphi E_{n v}=0
\end{aligned}
$$

From Equation (14), solving for $E^{*}$, we have

$$
E^{*}=\frac{(\rho+\varepsilon+y+\mu)}{(1-\theta) \sigma} I_{a}^{*}
$$

from Equation 15 solving for $I_{s}^{*}$ we have

$$
I_{s}^{*}=\frac{\theta(\rho+\varepsilon+y+\mu)+\varepsilon(1-\theta)}{(1-\theta)\left(e+\omega+\alpha_{1}+\mu\right)} I_{a}^{*}
$$

from Equation 16 , solving for $H_{m}^{*}$ we have

$$
H_{m}^{*}=\frac{\rho(1-\theta)\left(e+\omega+\alpha_{1}+\mu\right)+e(\theta(\rho+\varepsilon+y+\mu)+(1-\theta) \varepsilon)}{(x+i+\mu)(1-\theta)\left(e+\omega+\alpha_{1}+\mu\right)} I_{a}^{*} ;
$$

solving for $H_{c}^{*}$ from Equation (17) we have

$$
H_{c}^{*}=\left(\frac{x}{g+\alpha_{2}}\right)\left(\frac{\rho(1-\theta)\left(e+\omega+\alpha_{1}+\mu\right)+e(\theta(\rho+\varepsilon+y+\mu)+(1-\theta) \varepsilon)}{(x+i+\mu)(1-\theta)\left(e+\omega+\alpha_{1}+\mu\right)}\right) I_{a}^{*}
$$

solving Equation 18 for $R^{*}$ we have

$$
R^{*}=\frac{g}{\mu} H_{c}^{*}+\frac{i}{\mu} I H_{m}^{*}+\frac{y}{\mu} I_{a}^{*}+\frac{\omega}{\mu} I_{s}^{*}-0 \text { and }
$$

by solving Equation 20 for $E_{n v}^{*}$, we can get

$$
E_{n v}^{*}=\frac{a(1-\theta)\left(e+\omega+\alpha_{1}+\mu\right)+b[\theta(\rho+\varepsilon+y+\mu)+(1-\theta) \varepsilon]}{(1-\theta)\left(e+\omega+\alpha_{1}+\mu\right) \varphi} I_{a}^{*} .
$$

\subsection{Projection of Cases using Proposed Model}

The proposed modified SEIR model described in section 2.1 is used to project the number of COVID-19 related cases in Ethiopia. Projecting the number of active COVID-19 cases under the implementation of different NPIs with different adherence level is important for policy makers to be able to mitigate against the disease. Projecting the number of asymptomatic and symptomatic people is important, as these two numbers have huge roles in the spread of 
the disease. Further, knowing the expected number of people who may require treatment under intensive care (ICU cases) will greatly help to guide policy makers in preparation of manpower and medical facilities such as ventilators. Due to the very limited number of available ventilators in Ethiopia (below 1000 ventilators in total), knowing the projected number of individuals who need ICU under the implementation of different NPIs with varying adherence level of hygiene, physical distancing and mask wearing are important to save lives. Many studies showed that most of the critically ill patients with COVID-19 are of older age and have more co-morbidities than the non-critically ill patients. Hence, projection is done for all active case, symptomatic cases, asymptomatic cases, ICU cases, and death due to the disease using the proposed model.

In our model, common COVID-19 related parameters were obtained from previous studies (Table 1 . ப $^{2}$

Further, based on the 2016 Ethiopian demographic and health survey, $27.4 \%$ and $7.8 \%$ of the urban and rural population wash hands by soap, respectively. Thus, in the simulation, we assumed improved percentages of hygiene due to the awareness created by COVID-19 in both population settings. Due to differences in access to sanitation materials, lifestyle, cultural norms and other factors, adherence levels to the recommended NPIs are also different in the urban and rural populations. As a result, projection on the number of COVID-19 related cases is done separately for urban and rural populations of Ethiopia.

\subsection{Data}

In this study, data on daily number of COVID-19 cases, cumulative number of deaths, and number of critical patients was extracted from Ethiopian Public Health Institute website (www.ephi.gov.et/) and Ministry of Health official Twitter page(https://twitter.com/FMoHealth/) on daily basis. We used initial parameter (1)values describing the natural and clinical course of infection from published sources WHO (2020); Ngonghala et al. (2020); Ferguson et al. (2020); Kassa et al. (2020). The projection model considers daily number of cases in Ethiopia up to 210 days (October 08, 2020) since the first case of the disease was recorded.

\subsection{Considered Non-Pharmaceutical Interventions (NPIs)}

Based on the current evidence about COVID-19, implementing different NPIs are essential requirements for limiting the spread of the disease. The main objective of NPIs is to reduce the rate of transmission, thereby minimizing the size of the epidemic peak and delaying peak time, buying time for preparations in the healthcare system, and enabling the potential for vaccines and drugs to be developed, approved and used, Fong et al. (2020a); Ryu et al. (2020); Xiao et al. (2020); Flaxman et al. 2020); Lai et al. (2020). The NPIs considered in this study are physical-distancing, wearing face-masks and hygiene measures (hand washing)(see Table 2). We assume that implementing NPIs alone or in combination affects the rate of contact between uninfected people and infected people or objects. As a result, transmission probability of the virus from infected individuals or objects to the susceptible population is reduced.

We evaluated the impact of the three NPIs, alone or in combination, on the time and size of the peak by varying the adherence level to each of them.

\footnotetext{
${ }^{2}$ COVID-19 ICU:32.9\% died, $44.1 \%$ recovered https://www.bbc.com/news/uk-scotland-52653192
} 
Table 1: Description and values of initial model parameters 1

\begin{tabular}{|c|c|c|c|c|}
\hline \multirow{2}{*}{ Parameter } & \multirow{2}{*}{ Description } & \multicolumn{2}{|c|}{ Value } & \\
\hline & & Global-level & Ethiopia & \\
\hline$\gamma$ & force of infection & & estimated & \\
\hline$\beta_{1}$ & rate of disease transmission directly from human & .807 & & \\
\hline$\beta_{2}$ & rate of disease transmission from & & & \\
\hline & contaminated environment & 0.4 & & \\
\hline$\eta_{a}$ & relative infectiousness of person in $I_{a}$ & 0.5 & & \\
\hline$\epsilon$ & proportion of the exposed that goes to $I_{a}$ & 0.2 & & \\
\hline$\theta$ & proportion of the exposed transfer to $I_{s}$ & 0.8 & & \\
\hline$\sigma$ & rate at which exposed individuals are detected and move to $I$ & 0.2 & & \\
\hline$\alpha_{2}$ & Disease induced death rate & 0.15 & 0.015 & \\
\hline $\mathrm{a}$ & Shedding rate from the asymptomatic Infectious class & $1 / 8$ & & Ferguson et al. \\
\hline $\mathrm{b}$ & Shedding rate from the symptomatic infectious class & $1 / 10$ & & \\
\hline$\varphi$ & Virus decays from the environment & $1 / 4$ & & \\
\hline$\varepsilon$ & rate of $I_{a}$ develop symp. and going to $I_{s}$ & 0.4 & & \\
\hline e & rate of detected from $I_{s}$ going to $I H_{m}$ & 0,8 & & \\
\hline$\rho$ & rate of detection and isolating or go to $I H_{m}$ & 0.4 & & \\
\hline $\mathrm{y}$ & Rate of recovery from $I_{a}$ & 0.2 & & \\
\hline$\omega$ & Rate of recovery from $I_{s}$ & 0.4 & & \\
\hline $\mathrm{x}$ & Rate of transfer from $I H_{m}$ to $H_{c}$ & 0.08 & 0.01 & \\
\hline$\alpha_{1}$ & Death Rate of transfer from $I_{s}$ & 0.1 & & \\
\hline $\mathrm{g}$ & Rate of Recovery after getting critical & 0.02 & 0.08 & \\
\hline $\mathrm{i}$ & Rate of Recovery after getting mild & 0.4 & 0.6 & \\
\hline$\mu$ & Natural death rate & 0.0076 & 0.0064 & Organ \\
\hline$\pi$ & Natural birth rate & 0.018 & 0.03 & \\
\hline
\end{tabular}


Table 2: Summary of considered intervention mechanisms and their effect on reducing the spread of COVID19 based on existing literature

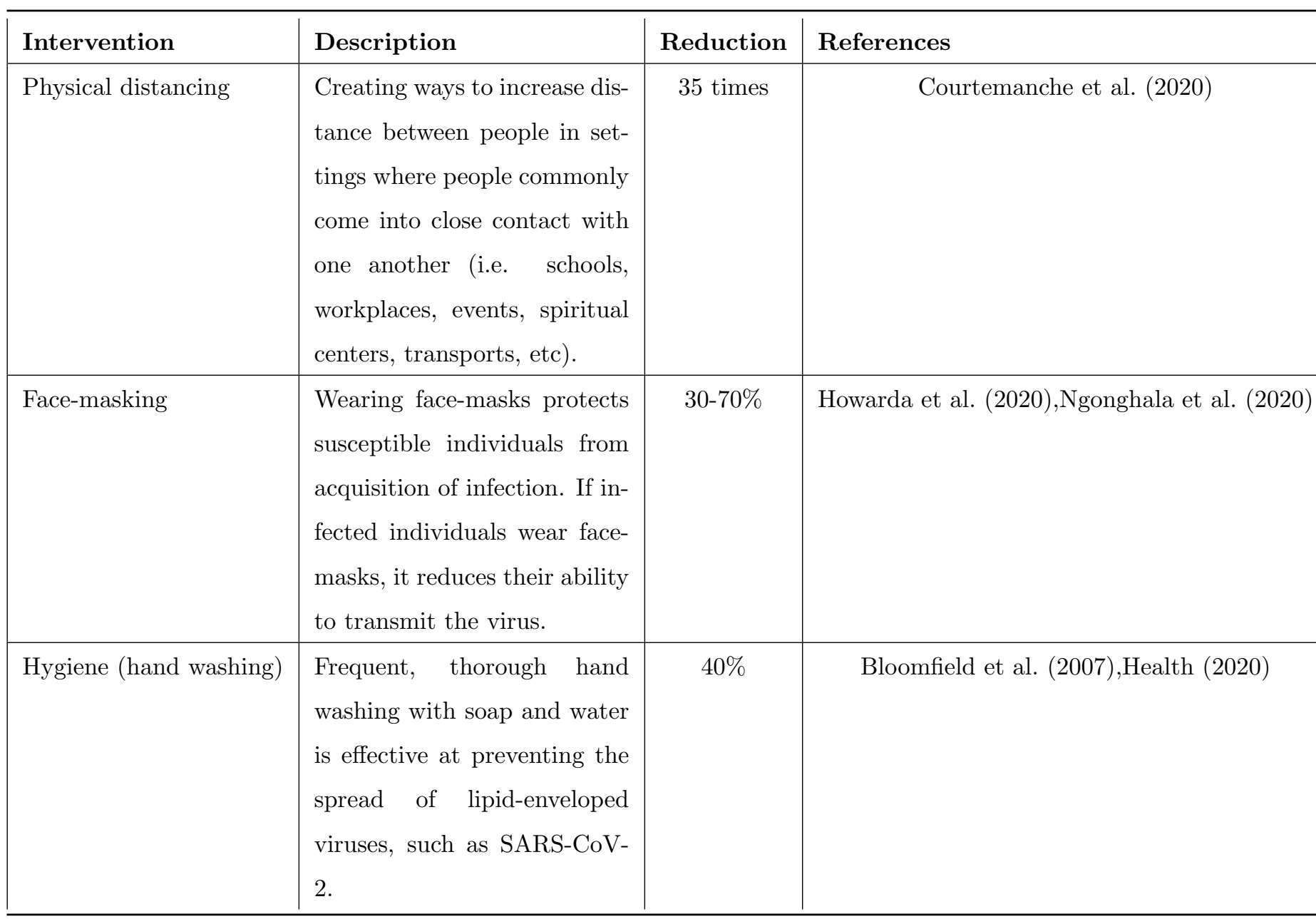

\section{Results: Projection of number of COVID-19 Cases in Ethiopia}

\subsection{Projection of All Active Cases}

By fixing the adherence levels to hygiene to $30 \%$ and $40 \%$ of the urban population based on the aforementioned reasons, the projected number of active COVID-19 cases was obtained by varying the percentages of social distancing and face mask coverage. Figure 2 presents the projected number of active COVID-19 cases with varying adherence levels of NPIs.

At $15 \%$ face mask coverage and with $10 \%$ of the population implementing social distancing measures, a $10 \%$ increase in hygiene will decrease the number of cases by 200,000 and delay the peak time. For a given implementation of wearing a face mask and hygiene, improving the percentage of social distancing by $10 \%$ will reduce the number 


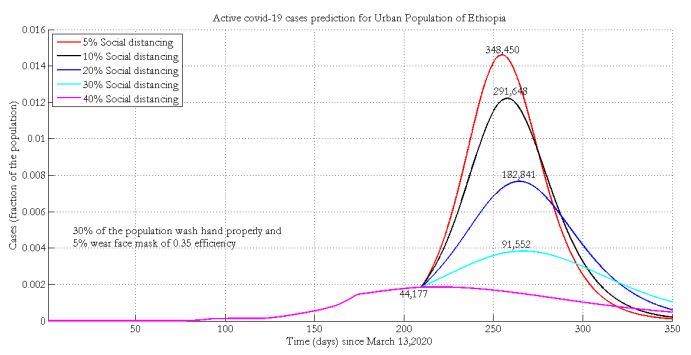

(a) $30 \%$ Hygiene and $5 \%$ face mask

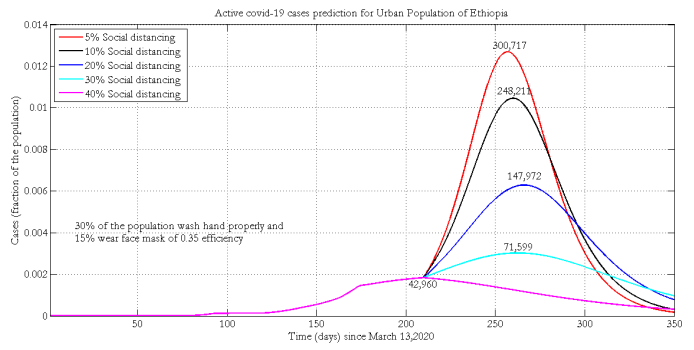

(c) $30 \%$ Hygiene and $15 \%$ face mask

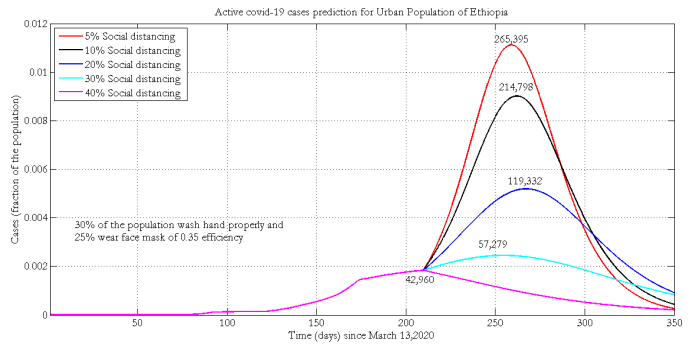

(e) $30 \%$ Hygiene and $25 \%$ face mask

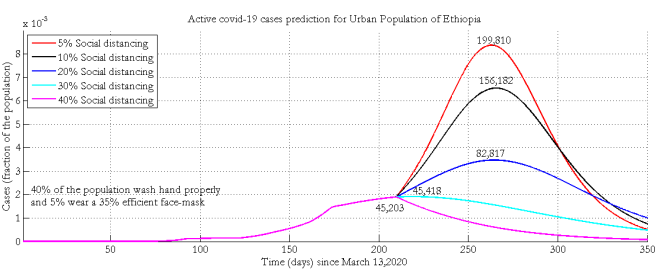

(b) $40 \%$ Hygiene and $5 \%$ face mask

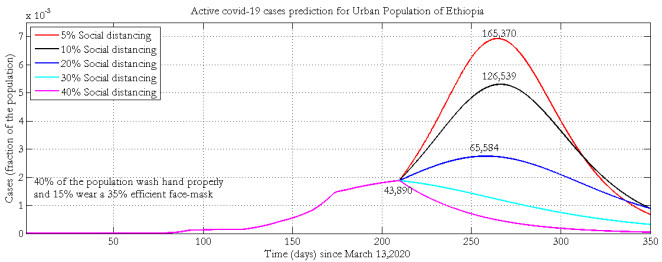

(d) $40 \%$ Hygiene and $15 \%$ face mask

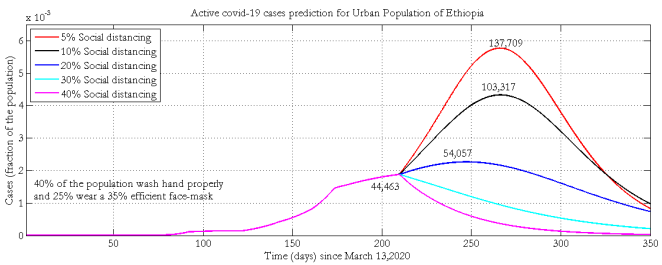

(f) $40 \%$ Hygiene and $25 \%$ face mask

Figure 2: Projection of active COVID-19 cases in urban population of Ethiopia when only 5\% (Figure 2a and $2 \mathrm{~b}$ ), $15 \%$ (Figure 2c 2d) and 25\% (Figure 2e 2f) of the population wear face masks with $35 \%$ efficacy 
of new COVID-19 cases by one-fifth. Increasing the number of people who wear face mask a by $10 \%$ will save on average 25,000 people from being infected by the disease (Figure 2).

In the rural parts of Ethiopia, physical distancing is a custom and part of the life style. This custom greatly minimizes the spread of the disease that is caused by contact and contaminated environment. Furthermore, due to low coverage of road accessibility (accessibility index=22\%), in our simulation we assumed $20 \%$ of the population are protected until the end of the pandemic World-Bank (2016). Further, based on 2016 EDHS, 7.8\% of the rural population wash their hands using soap. Figure 3 depicts the projected number of active cases under the implementations of varying physical distancing measures and wearing of face masks. In rural settings, people use home-made masks, and we assumed the efficacy of the masks is 0.2 .

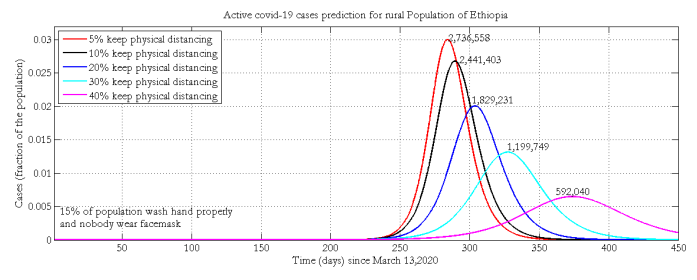

(a) $15 \%$ hygiene and nobody wear face mask

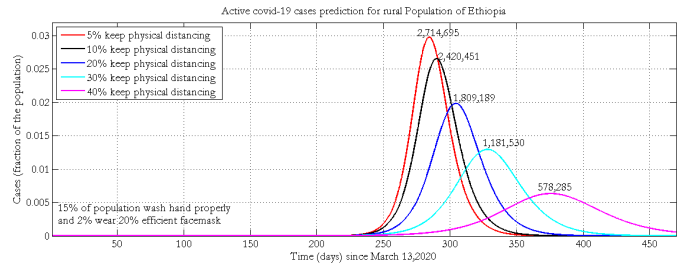

(c) $15 \%$ hygiene and $2 \%$ wear face mask

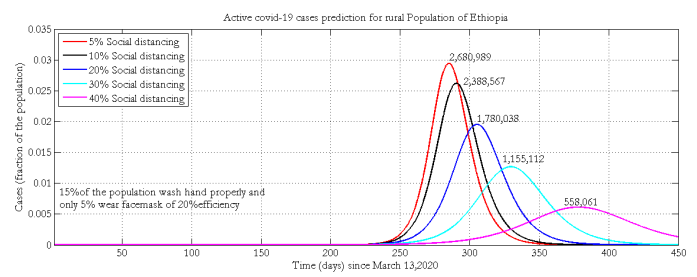

(e) $15 \%$ hygiene and $5 \%$ wear face mask

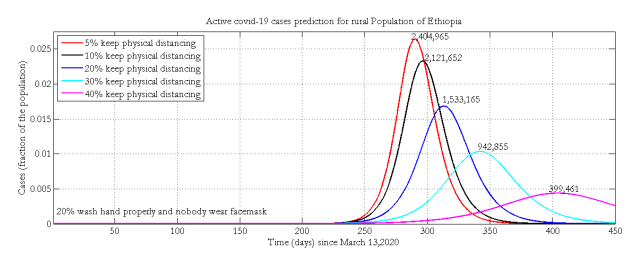

(b) $20 \%$ hygiene and nobody wear face mask

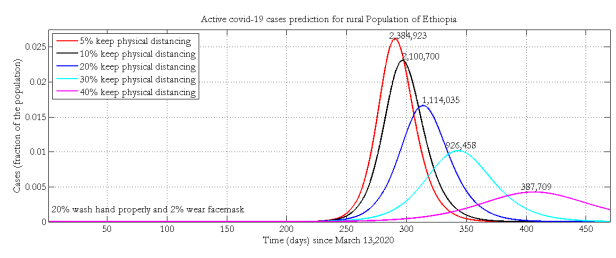

(d) $20 \%$ hygiene and $2 \%$ wear face mask

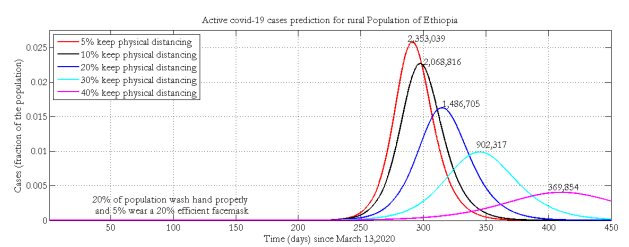

(f) $20 \%$ hygiene and $5 \%$ wear face mask

Figure 3: Projection of active COVID-19 cases in rural population of Ethiopia with 0\% (Figure 3a.Figure 3b) $2 \%$ (Figure 3c Figure 3d) 5\% (Figure 3e,Figure 3f) wear face masks with $20 \%$ efficiency.

Keeping adherence to face mask wearing and physical distancing constant, a $5 \%$ increase in hygiene will decrease the number of cases by 200,000 and shift the peak time to the future nearly by one month (Figure 3 . For a given implementation of face mask wearing and hygiene, improving the percentage of physical distancing by $10 \%$ will reduce the number of new COVID-19 cases by one-fourth. 


\subsection{Projection of Symptomatic and Asymptomatic Cases}

The following projected number of symptomatic (broken line) and asymptomatic (solid line) cases were simulated by assuming $30 \%$ and $40 \%$ of the urban population wash hands with soap, with different face mask coverage $(5 \%, 15 \%$ and $25 \%$ ) with $35 \%$ mask efficacy.

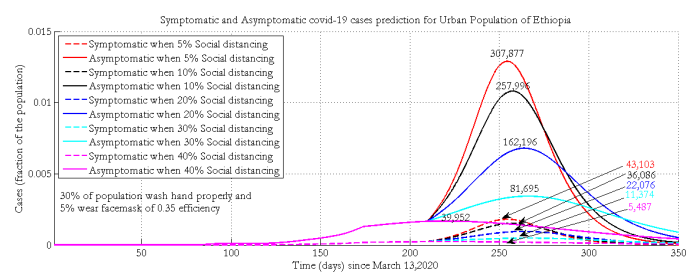

(a) $30 \%$ Hygiene and $5 \%$ face mask

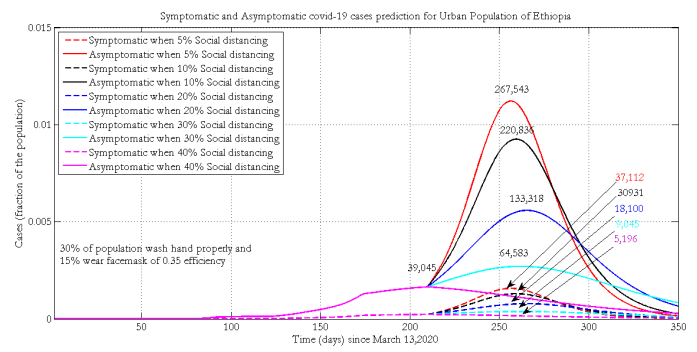

(c) $30 \%$ Hygiene and $15 \%$ face mask

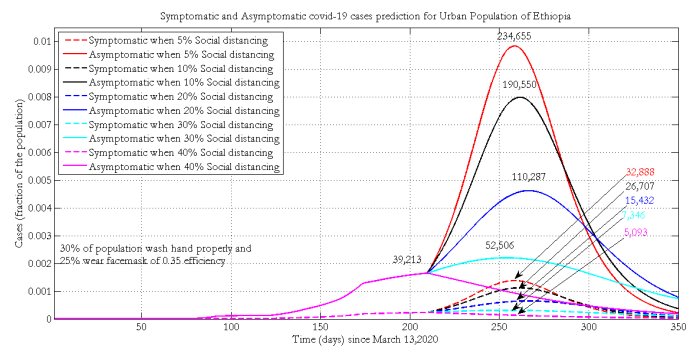

(e) $30 \%$ Hygiene and $25 \%$ face mask

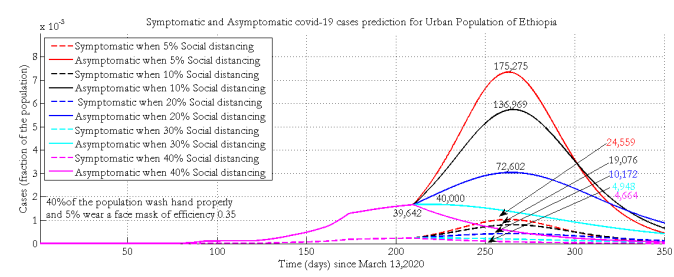

(b) $40 \%$ Hygiene and $5 \%$ face mask

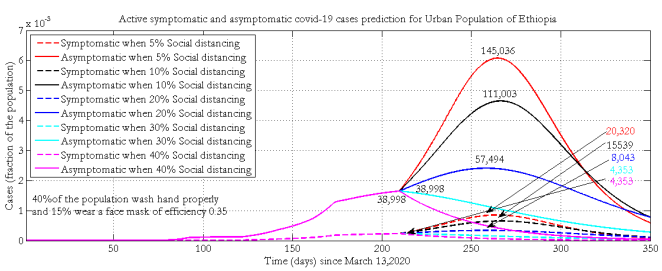

(d) $40 \%$ Hygiene and $15 \%$ face mask

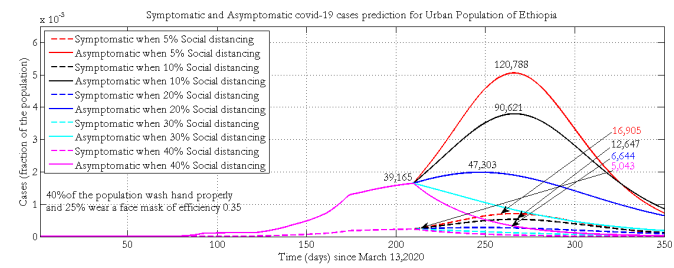

(f) $40 \%$ Hygiene and $25 \%$ face mask

Figure 4: Projection of symptomatic and asymptomatic infectious cases in urban populations of Ethiopia

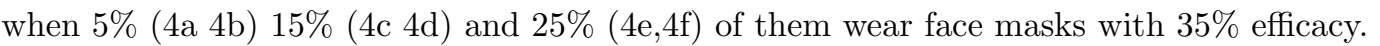

When $40 \%$ of the urban population wash hands with soap or keep hands clean using sanitizers, $25 \%$ wear face masks, improving the implementation of physical distancing greatly reduces both symptomatic and asymptomatic infections. By increasing the percentage of physical distancing in the population from $10 \%$ to $20 \%$, the peak size will decrease by half for both infection compartments (Figure 4).

For a given coverage of face mask wearing and implementing physical distancing, improving hygiene by $10 \%$, highly reduces the number of active cases and delays the peak time of the pandemic. For both hygiene scenarios, for a given level of face mask wearing, increasing adherence to physical distancing profoundly reduces the number of symptomatic and asymptomatic cases. A maximum number of asymptomatic infections will be observed if there 
is poor implementation of physical distancing and face mask wearing. Regardless of other NPIs, COVID-19 will be suppressed if $40 \%$ of the population implements physical distancing properly.

Figure 5 presents the projected number of symptomatic (broken line) and asymptomatic (solid line) cases in rural Ethiopia by varying the level of face mask wearing and physical distancing while fixing hygiene practice in the community at $15 \%$ and $20 \%$. Increasing proper hand washing behaviour from $15 \%$ to $20 \%$ could shift the peak by around 2 months. Implementing physical distancing, hygiene, and face mask wearing at 40\%, 20\% and 5\% levels, respectively, helps to control the pandemic under the Ethiopian health system capacity and shifts the peak forward to 2021. At a given level of implementation of hand washing and wearing of face masks, increasing the physical distancing by $10 \%$ could shift the peak time by around 40 days (Figure 5 .

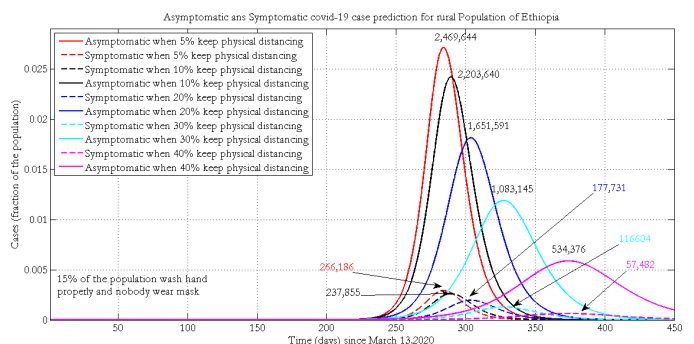

(a) $15 \%$ hygiene and nobody wear a face mask

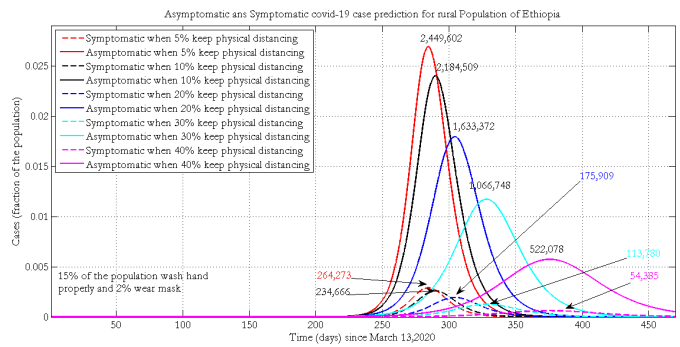

(c) $15 \%$ hygiene and $2 \%$ wear a face mask

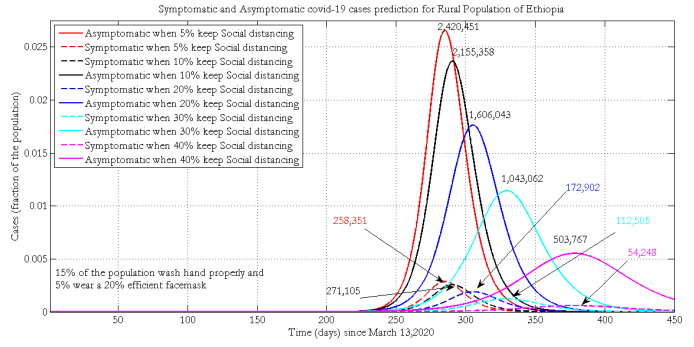

(e) $15 \%$ hygiene and $5 \%$ wear a face mask

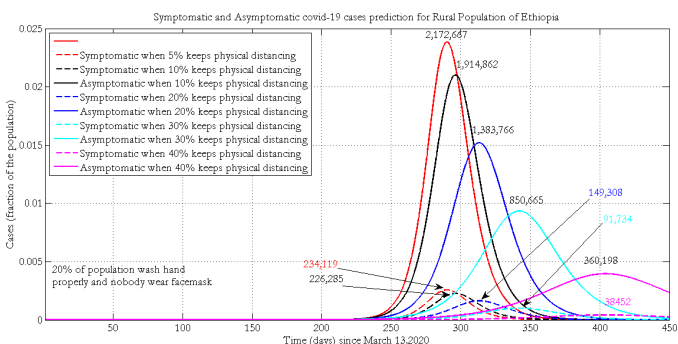

(b) $20 \%$ hygiene and nobody wear face mask

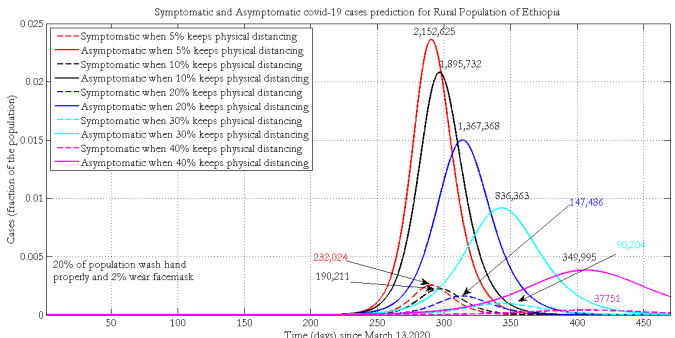

(d) $20 \%$ hygiene and $2 \%$ wear face mask

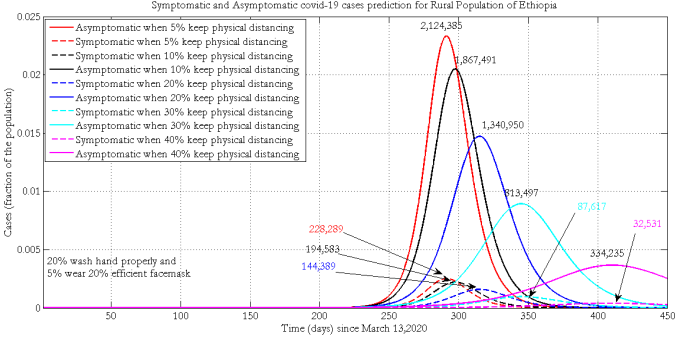

(f) $20 \%$ hygiene and $5 \%$ wear face mask

Figure 5: Projection of symptomatic and asymptomatic infectious cases in rural population of Ethiopia when 0\%(Figure 5a, Figure 5b 2\% (Figure 5c Figure 5d) 5\%(Figure 5e,Figure 5f) of the rural population properly wear a face mask 


\subsection{Projection of Critical Cases}

Figure 6 presents the projected number of ICU cases by keeping the hygiene coverage constant (at 30\%and 40\%) and varying adherence to physical distancing and wearing of face masks at different levels in urban population of Ethiopia.

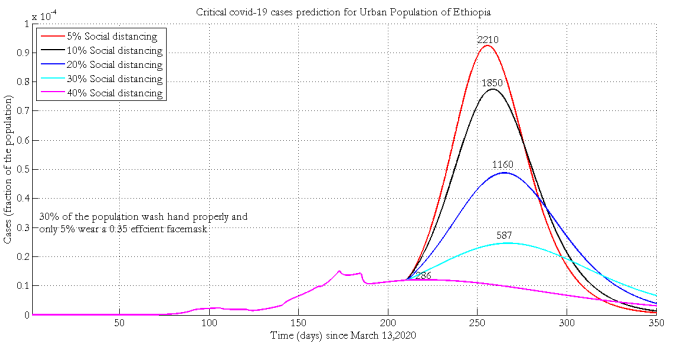

(a) $30 \%$ hygiene and $5 \%$ face-mask coverage

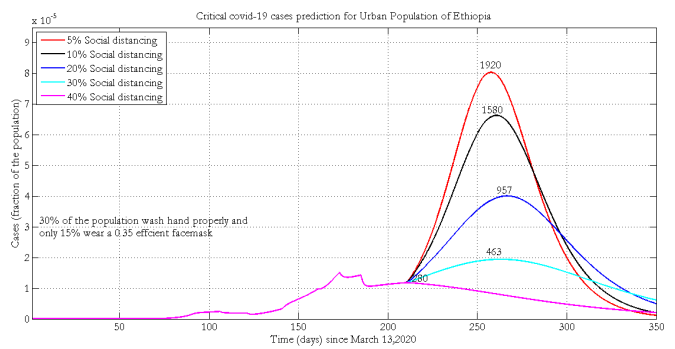

(c) $30 \%$ hygiene and $15 \%$ face-mask coverage

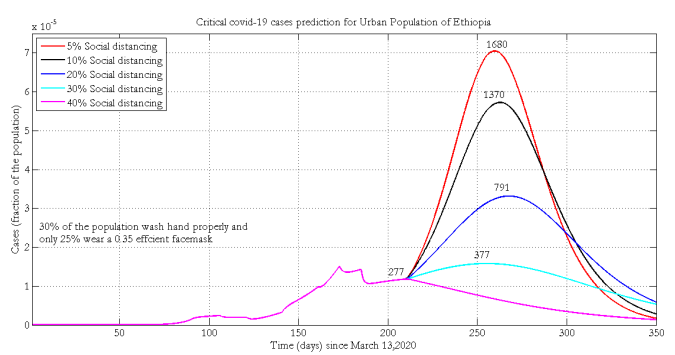

(e) $30 \%$ hygiene and $25 \%$ face-mask coverage

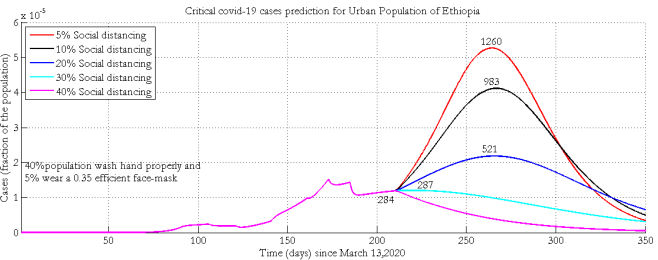

(b) $40 \%$ hygiene and $5 \%$ face-mask coverage

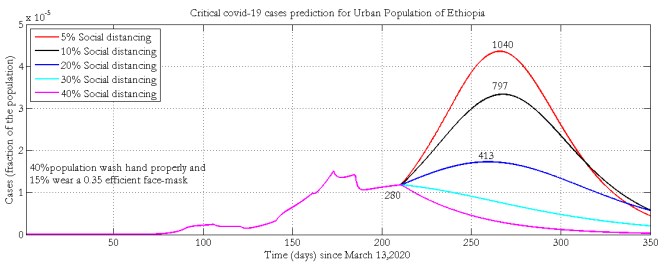

(d) $40 \%$ hygiene and $15 \%$ face-mask coverage

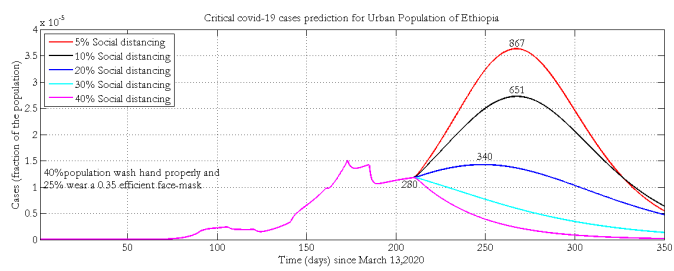

(f) $40 \%$ hygiene and $25 \%$ face-mask coverage

Figure 6: Projection number of urban population who will need intensive care when 5\%(Figure 6a Figure 6b) $15 \%$ (Figure 6c,Figure 6d) and 25\% (Figure 6e, Figure 6f) of them wear face mask

If $15 \%$ of the population wears masks and $30 \%$ implement physical distancing, improving hygiene by $10 \%$ could reduce the number of ICU cases from 463 to 280 (Figure6). Proper implementation of wearing face masks and hand washing could flatten the curve. However, to bring critical cases under the Ethiopian health care capacity, people should also practice social distancing.

It is not too late to suppress the disease if strict implementation of NPIs is practiced (40\% hand wash, $40 \%$ physical distancing and 25\% wearing masks) in all urban populations of Ethiopia. 
In the rural population the projected number of critical cases could exceed the available number of ventilators in our health system (Figure 7). To contain critical cases, people should strictly implement the three NPIs or the government should enforce additional public health measures. Since the time of the peak in rural populations is different from that of urban populations, the health system can make preparations for the transport system to bring these critical cases to heath facilities in urban centers. The two extreme scenarios show that improving hygiene by $5 \%$ and physical distancing by $40 \%$ could reduce the total?at peak time? number of critical cases from 2,361 to 1,569 (assuming 5\% wear masks). Strict implementation of the three NPIs will shift the peak time forward by three months (Figure 7).

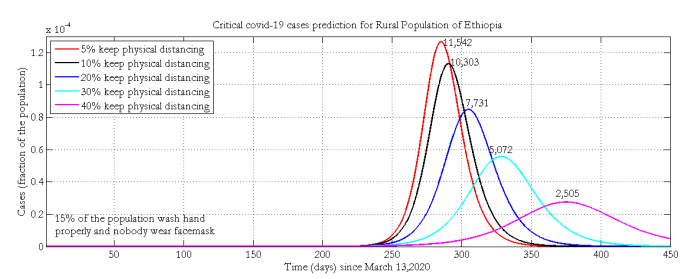

(a) $15 \%$ hygiene and nobody wear face mask

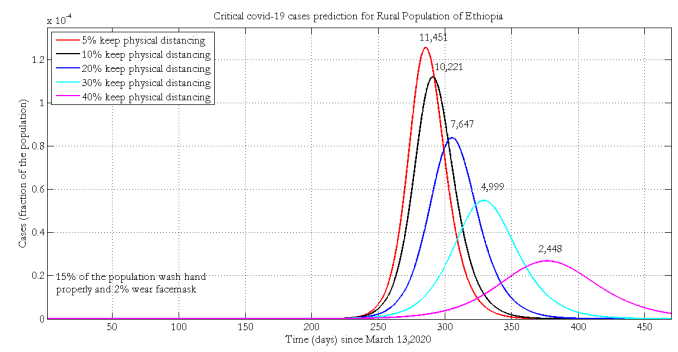

(c) $15 \%$ hygiene and $2 \%$ wear face mask

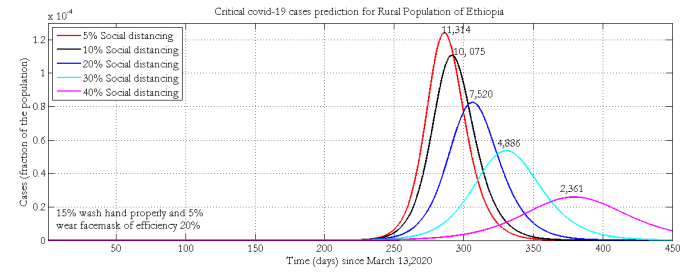

(e) $15 \%$ hygiene and $5 \%$ face mask

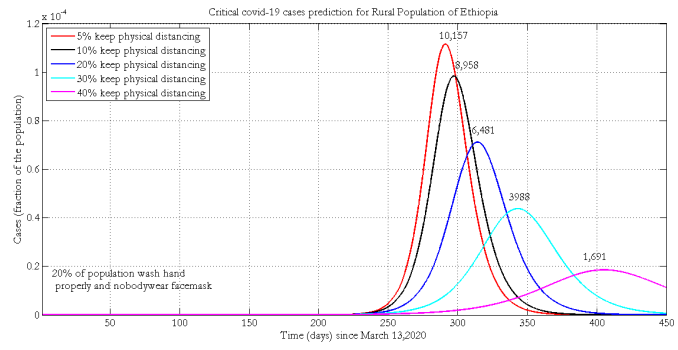

(b) $20 \%$ hygiene and nobody wear face mask

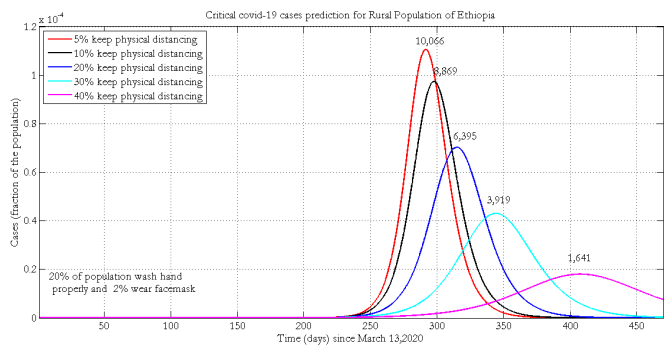

(d) $20 \%$ hygiene and $2 \%$ wear face mask

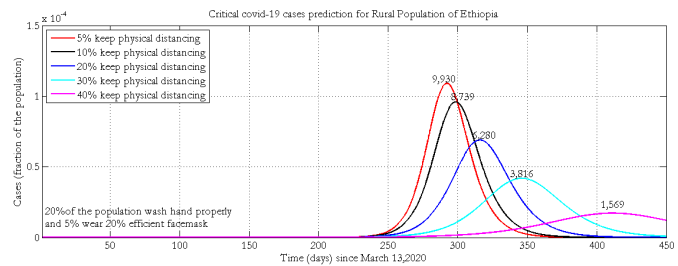

(f) $20 \%$ hygiene and $5 \%$ wear face mask

Figure 7: Projection of intence care or critical cases in the rural population of Ethiopia when 0\% (Figure 7a. Figure 7b 2\% (Figure 7c, Figure 7d) 5\%(Figure 7e,Figure 7f) of the population are wearing facemask with $20 \%$ efficacy. 


\subsection{Projection of Cumulative Death Due to COVID-19}

Figure 8 presents the projected number of deaths due to COVID-19 under different scenarios in urban populations of Ethiopia.

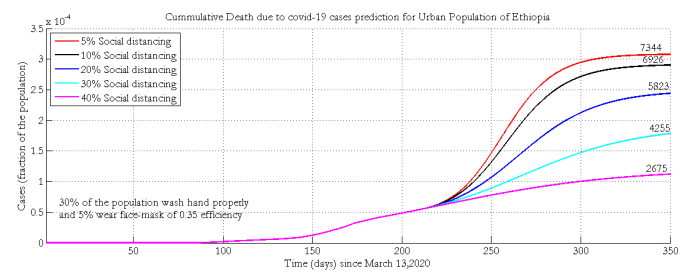

(a) $30 \%$ hygiene and $5 \%$ face mask coverage

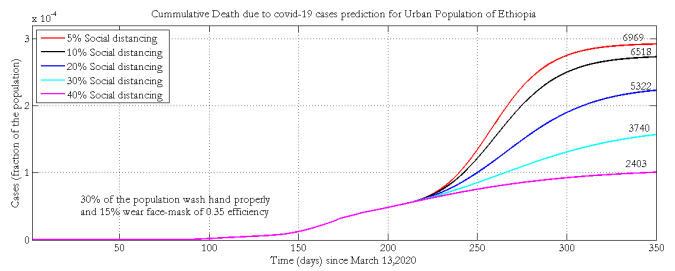

(c) $30 \%$ hygiene and $15 \%$ face mask coverage

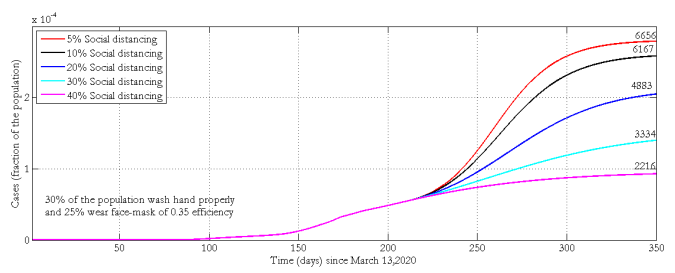

(e) $30 \%$ hygiene and $25 \%$ face mask coverage

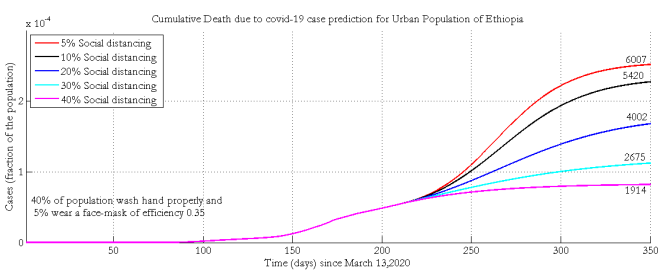

(b) $40 \%$ hygiene and $5 \%$ face mask coverage

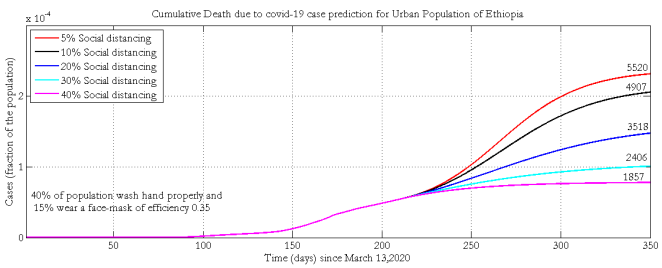

(d) $40 \%$ hygiene and $15 \%$ face mask coverage

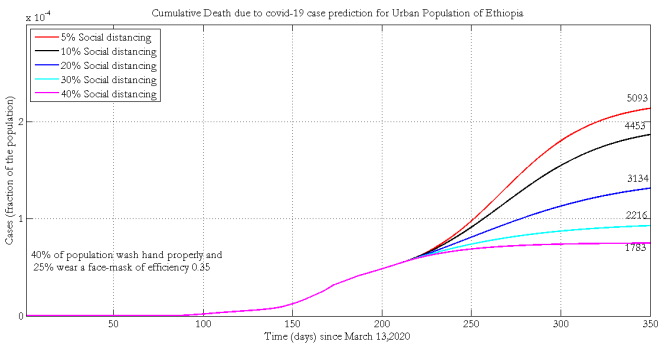

(f) $40 \%$ hygiene and $25 \%$ face mask coverage

Figure 8: Projection of deaths under the implementation of different NPIs when 5\%(Figure 8a, Figure 8b) 15\% (Figure 8c Figure 8d) and 25\% (Figure 8e, Figure 8f of them wear face masks properly.

The number of deaths due to COVID-19 could be reduced if more than $40 \%$ of the populations practice physical distancing. The projected number of cumulative deaths under the implementation of different adherence levels of physical distancing and face mask wearing at fixed (15\% and 20\%) levels of hygiene are presented in Figure 9

Practicing recommended NPIs properly (40\% physical distancing 5\% face mask and 20\% proper hand washing) could greatly reduce the number of death due to COVID-19. Table 3 and Table 4 presents summary of the projected number of active COVID-19 and ICU cases under different adherence levels of hygiene, wearing-masks and physicaldistancing in the urban and rural population of Ethiopia, respectively. 


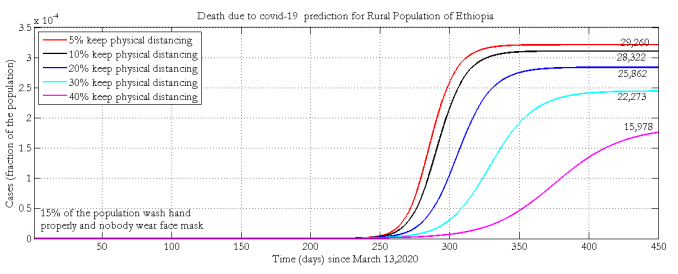

(a) $15 \%$ hygiene and nobody wear face mask

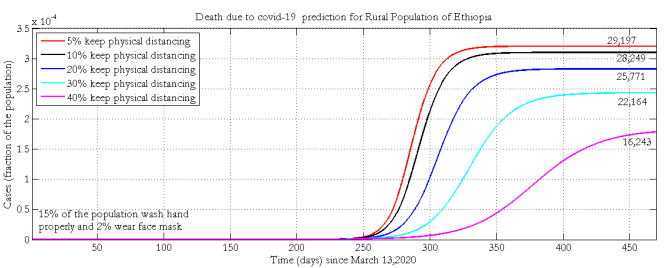

(c) $15 \%$ hygiene and $2 \%$ wear face mask

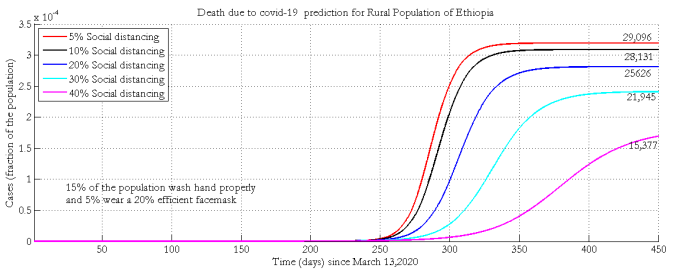

(e) $15 \%$ hygiene and $5 \%$ wear face mask

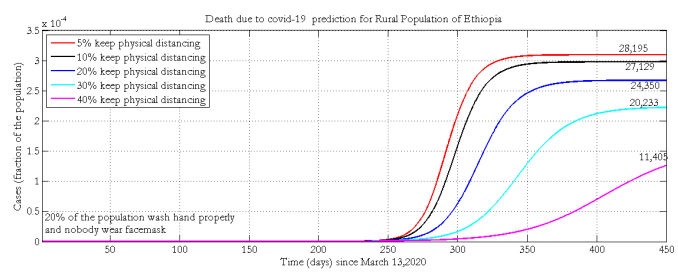

(b) $20 \%$ hygiene and nobody wear face mask

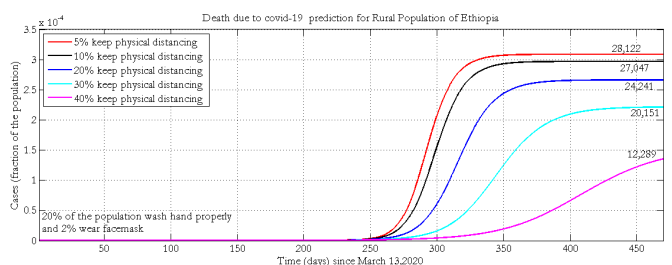

(d) $20 \%$ hygiene and $15 \%$ wear face mask

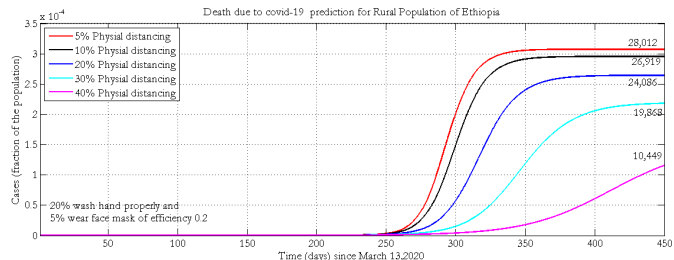

(f) $20 \%$ hygiene and $5 \%$ wear face mask

Figure 9: Projected number of cumulative death due to covid-19 in rural Ethiopia when nobody (Figure 9a,Figure 9b) 2\% (Figure 9c, Figure 9d) 5\%(Figure 9e,Figure 9f) of the population wear face mask of 0.2 efficiency. 
Table 3: Summary of predicted number of active Covid-19 and ICU cases (in thousands) under different adherence levels of wearing-masks and physical-distancing in the Urban population of Ethiopia.

\begin{tabular}{|c|c|c|c|c|c|c|c|c|c|c|c|c|}
\hline \multirow[t]{4}{*}{ Distancing } & \multicolumn{12}{|c|}{ Percentage of population who wear face masks of efficiency $35 \%$, Hygiene $=30 \%$} \\
\hline & \multicolumn{6}{|c|}{ Projected Number of all active Cases } & \multicolumn{6}{|c|}{ Projected number of active ICU cases } \\
\hline & \multicolumn{2}{|c|}{5} & \multicolumn{2}{|c|}{15} & \multicolumn{2}{|c|}{25} & \multicolumn{2}{|r|}{5} & \multicolumn{2}{|c|}{15} & \multicolumn{2}{|c|}{25} \\
\hline & Size & Period & Size & Period & Size & Period & Size & Period & Size & Period & Size & Period \\
\hline 5 & 348 & Nov. & 300 & Nov. & 265 & Nov. & 2.21 & Nov. & 1.92 & Nov. & 1.68 & Nov. \\
\hline 10 & 292 & Nov. & 248 & Nov. & 214 & Nov. & 1.85 & Nov. & 1.58 & Nov. & 1.37 & Dec. \\
\hline 20 & 183 & Dec. & 147 & Dec. & 119 & Dec. & 1.16 & Dec. & 0.95 & Dec. & 0.79 & Dec. \\
\hline 30 & 91 & Dec. & 71 & Dec. & 57 & Nov. & 0.5 & Dec. & 0.46 & Dec. & 0.37 & Nov. \\
\hline \multirow[t]{2}{*}{40} & 44 & Oct. & 42 & Oct. & 42 & Oct. & 0.2 & Oct. & 0.2 & Oct. & 0.27 & Nov. \\
\hline & \multicolumn{12}{|c|}{ Percentage of population who wear-facemasks of efficiency $35 \%$, Hygiene $=40 \%$} \\
\hline 5 & 199 & Dec. & 165 & Dec. & 137 & Dec. & 1.26 & Dec. & 1.04 & Dec. & 0.8 & Dec. \\
\hline 10 & 156 & Dec. & 126 & Dec. & 103 & Dec. & 0.9 & Dec. & 0.7 & Dec. & 0.6 & Dec. \\
\hline 20 & 82 & Dec. & 65 & Nov. & 57 & Nov. & 0.5 & Dec. & 0.4 & Nov. & 0.3 & Nov. \\
\hline 30 & 45.4 & Oct. & 44 & Oct. & 44 & Oct. & 0.2 & Oct. & 0.2 & Oct. & 0.2 & Oct. \\
\hline 40 & 45.2 & Oct. & 44 & Oct. & 44 & oct. & 0.2 & Oct. & 0.2 & Oct. & 0.2 & Oct. \\
\hline
\end{tabular}


Table 4: Summary of predicted number of active Covid-19 and ICU cases (in thousands) under different adherence levels of wearing-masks and social-distancing in the rural population of Ethiopia

\begin{tabular}{|c|c|c|c|c|c|c|c|c|c|c|c|c|}
\hline \multirow[t]{4}{*}{ Face-masking } & \multicolumn{12}{|c|}{ Percentage of population who wear-facemasks of efficiency $20 \%$, Hygiene $=15 \%$} \\
\hline & \multicolumn{6}{|c|}{ Projected Number of all active Cases } & \multicolumn{6}{|c|}{ Projected number of active ICU cases } \\
\hline & \multicolumn{2}{|r|}{0} & \multicolumn{2}{|r|}{2} & \multicolumn{2}{|r|}{5} & \multicolumn{2}{|r|}{0} & \multicolumn{2}{|r|}{2} & \multicolumn{2}{|r|}{5} \\
\hline & Size & Period & Size & Period & Size & Period & Size & Period & Size & Period & Size & Period \\
\hline 5 & 2736 & Dec. & 2714 & Dec. & 2680 & Dec. & 11 & Dec & 11 & Dec & 11 & Dec \\
\hline 10 & 2441 & Dec. & 2420 & Dec. & 2388 & Dec & 10 & Dec. & 10 & Dec. & 10 & Dec. \\
\hline 20 & 1829 & Jan 21 & 1809 & Jan 21 & 1780 & Jan. 21 & 7 & Jan 21 & 7 & Jan 21 & 7 & Jan 21 \\
\hline 30 & 1199 & Feb. 21 & 1181 & Feb.21 & 1155 & Feb. 21 & 5 & Feb 21 & 5 & Feb 21 & 5 & Feb.21 \\
\hline \multirow[t]{2}{*}{40} & 592 & Mar 21 & 578 & Mar. 21 & 558 & Mar. 21 & 2 & Mar 21 & 2 & Mar 21 & 2 & Mar 21 \\
\hline & \multicolumn{12}{|c|}{ Hygiene improved from $15 \%$ to $20 \%$} \\
\hline 5 & 2404 & Dec. & 2384 & Dec. & 2353 & Dec. & 10 & Dec. & 10 & Dec. & 9 & Dec. \\
\hline 10 & 2121 & Jan 21 & 2100 & Jan 21 & 2069 & Jan 21 & 8 & Jan 21 & 8 & Jan 21 & 8 & Jan 21 \\
\hline 20 & 1533 & Jan 21 & 1514 & Jan 21 & 1485 & Jan 21 & 6 & Jan 21 & 6 & Jan 21 & 6 & Jan 21 \\
\hline 30 & 942 & Feb 21 & 926 & Feb 21 & 902 & Feb 21 & 3 & Feb 21 & 3 & Feb 21 & 3 & Feb 21 \\
\hline 40 & 399 & Mar 21 & 387 & Mar 21 & 370 & Mar 21 & 1 & Mar 21 & 1 & Mar 21 & 1 & Mar 21 \\
\hline
\end{tabular}




\subsection{The Basic Reproductive Number}

The basic reproductive number $\left(R_{o}\right)$ measures the degree of spread of SARS-CoV-2. If this number is greater than one, the disease will spread out if additional public health measures are not taken.

Table 5 and 6 presents estimated $R_{o}$ at different time points by taking into account enforced NPIs since the onset of the disease in urban and rural Ethiopia, respectively. This number was higher in the first and $10^{t h}$ week of the pandemic in urban Ethiopia. Further, compared with the rural population, at the beginning of the pandemic $R_{o}$ was higher in the urban areas of Ethiopia.

Table 5: Estimated $R_{o}$ at different time points since the onset of COVID-19 in urban population of Ethiopia

\begin{tabular}{c|ccccccc}
\hline Days & 2-60days & 61-90days & 91-105 days & 106-120days & 121-140days & 141-160days & $161-172$ days \\
\hline$R_{o}$ & 0.605 & 0.95 & 0.46 & 0.409 & 0.58 & 0.53 & 0.568 \\
\hline \hline Days & 173 -184days & 185 -210days & & & & & \\
\hline$R_{o}$ & 0.45 & 0.45 & & & & &
\end{tabular}

Table 6: Estimated $R_{o}$ at different time points since the onset of COVID-19 in rural population of Ethiopia

\begin{tabular}{c|cccccc}
\hline days & 2 -60days & $61-90$ days & $91-105$ days & $106-120$ days & $121-140$ days & $141-160$ days \\
\hline$R_{o}$ & 0.61 & 0.5593 & 0.4645 & 0.41 & 0.58 & 0.54 \\
\hline \hline Days & $161-172$ days & $173-180$ days & $181-215$ days & & & \\
\hline$R_{o}$ & 0.53 & 0.40 & 0.43 & & &
\end{tabular}

Table 7 presents summary of $R_{o}$ under the implementation of different NPIs with difference adherence level in the urban population of Ethiopia. Improving adherence to different NPIs greatly reduces $R_{o}$ in both urban and rural population.

Table 7: Summary of $R_{o}$ under the implementations of different NPIs with difference adherence level in the urban population of Ethiopia.

\begin{tabular}{r|ccccccccccc}
\hline Face mask & \multicolumn{7}{|c}{ Percentage of Population who Practice Physical-distancing } \\
& \multicolumn{7}{|c|}{ Hygiene=30\% } & \multicolumn{3}{c}{ Hygiene=40\% } \\
& 5 & 10 & 20 & 30 & 40 & 5 & 10 & 20 & 30 & 40 \\
\hline 5 & 0.7057 & 0.6686 & 0.5943 & 0.5200 & 0.4457 & 0.6049 & 0.5731 & 0.5094 & 0.4457 & 0.3820 \\
15 & 0.6554 & 0.6209 & 0.5520 & 0.4830 & 0.4140 & 0.5618 & 0.5322 & 0.4731 & 0.4140 & 0.3548 \\
25 & 0.6806 & 0.6448 & 0.5731 & 0.5015 & 0.4298 & 0.5834 & 0.5527 & 0.4912 & 0.4298 & 0.3684 \\
\hline
\end{tabular}




\subsection{Sensitivity Analysis}

Figure 10 shows that the model is particularly sensitive to physical distancing (SD), hand wash (HW) and direct human to human disease transmission rate $\left(\beta_{1}\right)$. Hand wash and physical distancing were inversely proportional to $R_{o}$, while $\beta_{1}$ has directly proportional to $R_{o}$. Parameters with relatively large PRCC values $(>0.5$ or $<-0.5)$ as

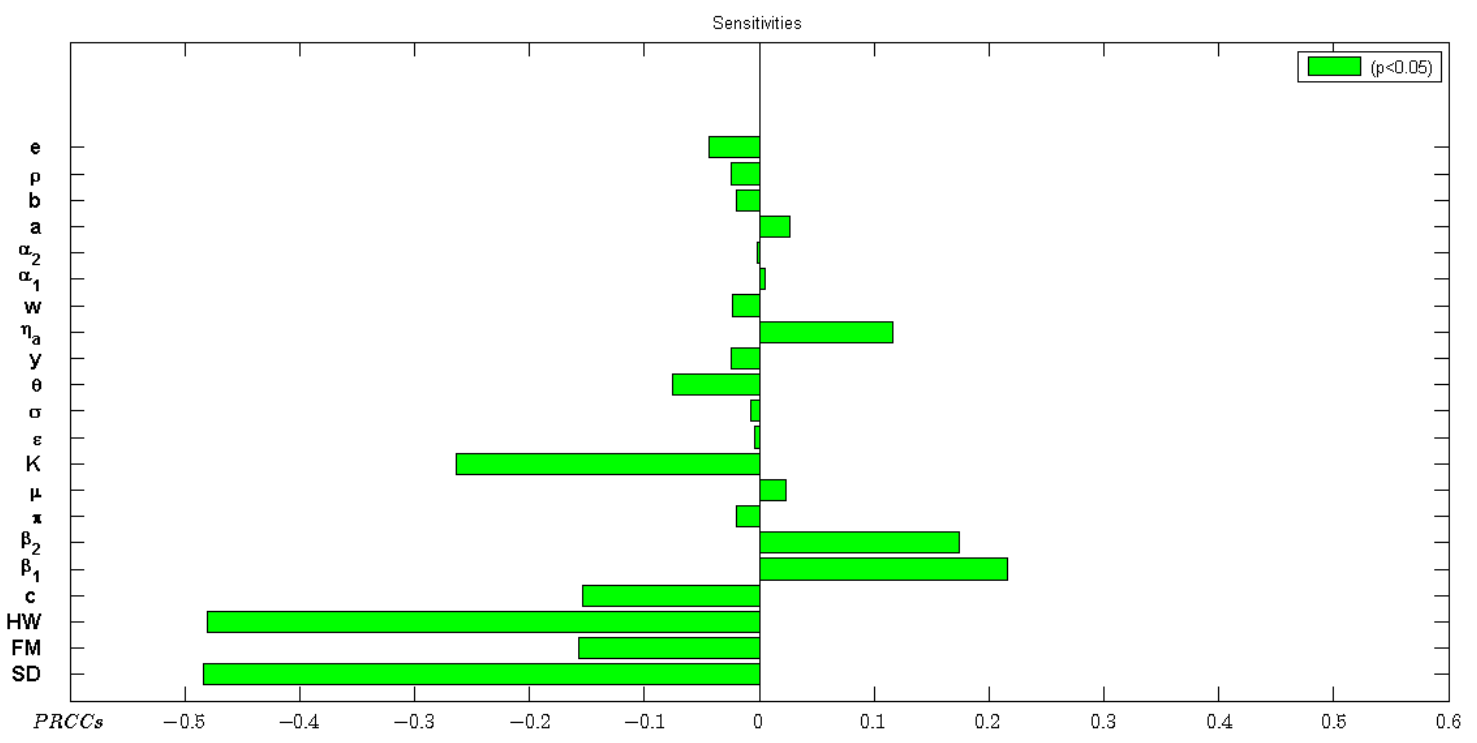

Figure 10: Sensitivity of parameters with respect to $R_{o}$

well as corresponding small p values $(<0.05)$ are considered as the most important parameters. Parameters whose PRCC value are close to +1 or -1 most strongly influence the model. A negative sign for PRCC indicates inverse relationship of the parameter with $R_{o}$.

To see whether one parameter depends on another, pairwise comparisons were carried out. The processes underlying the parameters physical distancing, hand washing and the proportion of the population who become symptomatic infectious, $\theta$, have the greatest potential of containing the epidemic if increased, whereas processes described by $\beta_{1}$ and $\epsilon$ and $\eta_{a}$ have the greatest potential of making the epidemic worse when increased (Figure 10.

In this respect, increasing physical distancing directly reduces $\beta_{1}$ as this lowers the likelihood of a susceptible individual getting in contact with a potentially infected individual. In addition, practicing good hygiene (such as regularly washing hands, using sanitizers to disinfect the infected environment, ventilation of rooms and avoiding touching the T-zones of the face) is associated with lowering the chance of contracting the virus from infected surfaces. Anything contrary to the above increases the likelihood of getting the infection through the two aforementioned routes. Moreover, improving hand wash practices will reduce viral contamination of the environment by infected individuals.

On the other hand, the processes underlying the parameters with negative PRCC have a potential to contain the number of cases when enhanced.

To ascertain whether the process described by those parameters are different or not, a pairwise comparison to 
significant parameters was undertaken. Table 8 presents the computed p-values for different pairs of significant parameters by accounting for the false discovery rate (FDR). The major question posed at this point is: Are the different pairs of significant parameters different after FDR adjustment? Based on the FDR adjusted p-values in Table 8, the compared pairs of parameters are rendered to be different if their p-value is less than 0.05 and not different otherwise.

Table 8: Summary of different pairs of significant parameters by accounting for the false discovery rate

\begin{tabular}{|c|c|c|c|c|c|c|c|c|c|c|c|}
\hline & $\mathrm{SD}$ & FM & HW & $\mathrm{c}$ & $\beta_{1}$ & $\varepsilon$ & $\theta$ & $\mathrm{y}$ & $\eta_{a}$ & $\mathrm{w}$ & e \\
\hline $\mathrm{SD}$ & & TRUE & FALSE & TRUE & TRUE & TRUE & TRUE & TRUE & TRUE & TRUE & TRUE \\
\hline FM & & & TRUE & FALSE & TRUE & TRUE & TRUE & FALSE & TRUE & TRUE & TRUE \\
\hline HW & & & & TRUE & TRUE & TRUE & TRUE & TRUE & TRUE & TRUE & TRUE \\
\hline $\mathrm{c}$ & & & & & TRUE & TRUE & TRUE & FALSE & TRUE & TRUE & TRUE \\
\hline$\beta_{1}$ & & & & & & TRUE & TRUE & TRUE & TRUE & TRUE & TRUE \\
\hline$\varepsilon$ & & & & & & & TRUE & TRUE & TRUE & TRUE & FALSE \\
\hline$\theta$ & & & & & & & & TRUE & TRUE & TRUE & TRUE \\
\hline $\mathrm{y}$ & & & & & & & & & TRUE & TRUE & TRUE \\
\hline$\eta_{a}$ & & & & & & & & & & TRUE & TRUE \\
\hline $\mathrm{w}$ & & & & & & & & & & & TRUE \\
\hline $\mathrm{e}$ & & & & & & & & & & & \\
\hline
\end{tabular}

Table 8 presents a summary of compared parameters, where "TRUE" indicates compared parameters are significantly different, and "FALSE" otherwise. The results show that more sensitive parameters are also significantly different (see Table 8 except for the pair rate of recovery of asymptomatic infectious individuals-appropriate use of face masking $y-F M$, recovery rate of symptomatic infectious individual-rate of going to isolation $w-e$, social distancing and hand washing $S D-H W$, respectively which may not necessarily be correlated.

\section{Discussion and Conclusion}

In this study, a modified SEIR model was developed to project the number of COVID-19 cases with different stage of the disease under the implementation of wearing face masks, hand washing and physical distancing with varying adherence levels in both urban and rural populations of Ethiopia. Unlike other mathematical modeling studies done in the context of Ethiopia Siraj et al. (2020); Alemneh and Telahun 2020); Takele (2020), this study provides projected number of all active cases, symptomatic and asymptomatic cases and ICU cases at the peak time of the pandemic. The projected number of people in each stage of the disease at the peak period and the time of the peak in urban and rural areas of Ethiopia helps the government to choose and enforce better intervention mechanisms.

Our projection shows that, in line with Howarda et al. (2020); Worby and H-H. (2020) improving the percentage 
of the population who wear face masks by $10 \%$ will protect around 100,000 more people from being infected by COVID-19 in the urban population. Regardless of face mask coverage and hygiene, the peak time of COVID-19 could be shifted to the future by two months if $40 \%$ of the population implements social distancing properly. Similar to our finding, the study by Siraj et al. (2020) demonstrated practicing physical distancing and wearing face masks delays the peak time of the pandemic.

In the rural population setting, implementing hygiene measures, physical distancing, and wearing face masks at $20 \%, 40 \%$, and $5 \%$, respectively, could shift the peak time into to 2021 . The projected number of active cases during the peak time could reach around 360,000 if $5 \%, 40 \%$, and $20 \%$ of the population wear masks, keep physical distancing and hand wash with soap, respectively. As social distancing is already a custom due to the life style in the rural population of Ethiopia, improving hygiene by $20 \%$ could help to decrease the number of cases by $2-3$ fold.

In the urban population setting, if $20 \%$ of the population implements physical distancing and $30 \%$ adopt hygiene measures, and $25 \%$ wear face masks the peak time of the pandemic will happen on December, 2020 with 119,000 estimated cases (Table 3). During the peak time of the pandemic, except for some scenarios (30\% physical distancing and above $\geq 15 \%$ face mask coverage), the projected number of ICU case are above the capacity of the Ethiopian health system, which needs the government attention (Table 3 ).

COVID-19 is a deadly virus for which vaccination or effective medical treatment is not yet available. Hence the government should focus on prevention of infection using NPI mechanisms. Low socioeconomic status and behavioral attitudes lead to closer interactions in congested households, hampering the successful implementation of social distancing measures. Lack or improper use of masks and use of low quality masks may exacerbate the spread of infection. It is therefore imperative that the prudent use of NPIs is implemented in Ethiopia to contain the pandemic. NPIs used in combination are able to decrease cases and fatalities due to Covid-19. Separate implementation of each of the NPIs shortens the peak time of the pandemic and the number of new cases will increase by two-fold. We considered the place of residence being urban and rural as a factor since adherence to the recommended NPIs and the life style and living conditions, varies greatly in urban and rural society of Ethiopia.

Similar to the urban population projection, projection of cases in the rural setting was done by modifying the model parameters in the context of rural population practices. Table 4 presented summary of projected active COVID-19 cases and ICU cases in rural population of Ethiopia under 30 scenarios. The results shows that, at the peak of the pandemic, the number of projected cases in rural population will be higher than the urban population.

\section{Conclusion}

The first COVID-19 case was detected on March 13, 2020 and since then the government of Ethiopia has applied different mitigation strategies. But the virus continues to spread. Since community transmission of the virus is established, temporally relaxing any of the three non-pharmaceutical interventions could cause a rebound of the number of new cases, potentially leading to a collapse of the health care system. Our findings confirm, in the context of Ethiopia, that suppression of COVID-19 could be achieved by the combined implementation of three public health measures: wearing of face masks, social distancing and hand hygiene. The most effective public health measures are 
found to be face mask wearing in urban population and social distancing in rural populations. To mitigate COVID-19, in the urban population of Ethiopia, strict implementation of all three NPIs are required. In addition to announcing public health measures, the government should work on proper enforcement of NPIs, and the public should properly practice public health measures for their own safety and well-being. Based on the projected results under different conditions, concerned stakeholders could recommend achievable NPIs for their implementation to policy makers.

\section{Limitation}

Studies on COVID-19 showed the distribution of cases strongly depend on age. The resources available could not get data on the number of cases by age group from concerned organizations in order to get age-structured predictions and to assess the impact of school closure on the pandemic. Further, the projection to the rural population setting is less reliable as the analysis has been constrained by different factors (i.e. exact number of incidences were not known, adherence to the implemented NPIs greatly vary across different regions of the country, estimated number of protected individuals assumed 20\%, and others). Further, the study did not account for under reporting of COVID-19 cases.

\section{Funding}

This work was supported by Addis Ababa University, Ethiopia, and as a sandpit project of the One Health Regional Network for the Horn of Africa (HORN) project. The HORN project is funded by UK Research and Innovation (UKRI) and the Global Challenges Research Fund (GCRF) from the Growing Research Capability call awarded to the University of Liverpool, UK. Funders had no role in the design, analysis and interpretation of the results.

\section{References}

Africa-CDC. Africa cdc. online https://africacdc.org/covid-19/, 32020.

H. Alemneh and G. Telahun. Mathematical modeling and optimal control analysis of covid-19 in ethiopia. $\underline{\text { Preprint }}$ on medRxiv DOI: $10.1101 / 2020.07 .23 .20160473,2020$.

R. M. Anderson and R. M. May. Population biology of infectious diseases: Part I., volume 280. Nature, 1979.

N. Banholzer, E. van Weenen, B. Kratzwald, A. Seeliger, D. Tschernutter, P. Bottrighi, A. Cenedese, J. P. Salles, W. Vach, and S. Feuerriegel. Impact of non-pharmaceutical interventions on documented cases of covid-19. online doi: https://doi.org/10.1101/2020.04.16.20062141, 42020.

K. Baye. Covid-19 prevention measures in ethiopia current realities and prospects. ppp, 62020. 
S. F. Bloomfield, A. Aiello, B. Cookson, C. O'Boyle, and E. L. Larson. The effectiveness of hand hygiene procedures in reducing the risks of infections in home and community settings including hand washing and alcohol-based hand sanitizers. American Journal of Infection Control, 35(10):s27-s64, 2007.

C. Courtemanche, J. Garuccio, A. Le, J. Pinkston, , and A. Yelowitz. Strong physical distancing measures in the united states reduced the covid-19 growth rate. preprint, 62020.

O. Diekmann, J. A. P. Heesterbeek, and J. A. J. Metz. On the definition and the computation of the basic reproduction ratio in models for infectious diseases in heterogeneous populations. Mathematical Biosciences, $28,1990$.

N. M. Ferguson, D. Laydon, G. Nedjati-Gilani, and N. Imai. Impact of non-pharmaceutical interventions (npis) to reduce covid-19 mortality and healthcare demand,. Imperial College COVID-19 Response Team., 2020.

S. Flaxman, S. Mishra, A. Gandy, H. J. T. Unwin, T. A. Mellan, H. Coupland, C. Whittaker, H. Zhu, T. Berah, J. W. Eaton, M. Monod, I. C. C.-. R. Team, A. C. Ghani, C. A. Donnelly, S. Riley, M. A. C. Vollmer, N. M. Ferguson, L. C. Okell, and S. Bhatt. Estimating the number of infections and the impact of non-pharmaceutical interventions on covid-19 in 11 european countries. Imperial College COVID-19 Response Team., 2020.

M. Fong, H. Gao, J. Y. Wong, J. Xiao, Y. S. Eunice, S. Ryu, and B. J. Cowling. Nonpharmaceutical measures for pandemic influenza in non health care settings-physical distancing measures. Emerg Infect Dis, 26:976-984, 2020a.

M. Fong, H. Gao, Y. Wong, J. Xiao, E. Shiu, S. Ryu, and B. Cowling. Nonpharmaceutical measures for pandemic influenza in non health care settings-physical distancing measures. Emerg Infect Dis, 26:976-984, 2020b.

T. Goshu, D.and Ferede, G. Diriba, and M. Ketema. Economic and welfare effects of covid-19 and responses in ethiopia: Initial insights. Ethiopian Economic Policy Research Institute, 2020.

N. J. Health. Hand-washing. online https://www.nationaljewish.org/patients-visitors/patient-info/importantupdates/coronavirus-information-and-resources/health-tips/prevention-tips/hand-washing, 62020.

J. Howarda, A. Huangb, Z. Lik, Z. Tufekcim, and V. Zdimal. Face masks against covid-19: An evidence review. doi: 10.20944/preprints202004.0203.v1, 2020.

E. P. H. Institute. Covid-19 pandemic preparedness and response in ethiopia, weekly bulletin. online https://www.ephi.gov.et/, 3 2020. www.ephi.gov.et.

B. Ivorra, M. Ferrández, and A. Vela-Pérez, M.and Ramos. Mathematical modeling of the spread of the coronavirus disease 2019 (covid-19) considering its particular characteristics. the case of china. preprint, 62020. http://www.doi.org/10.13140/RG.2.2.21543.29604.

S. M. Kassa, H. Njagarah, and Y. A. Terefe. Analysis of the mitigation strategies for covid-19: from a mathematical modelling perspective. preprint, 2020.

S. Kebede, N. Geda, B. Legesse, and B. Fekadu. Adoption of preventive practices for covid-19 in ethiopia. findings from a telephone-survey. Frontier, 2020. 
S. Lai, N. W. Ruktanonchai, L. Zhou, O. Prosper, W. Luo, and J. R. Floyd. Effect of non-pharmaceutical interventions to contain covid-19 in china. Nature, 2020.

Q. Li, X. Guan, P. , Wu, X. Wang, L. Zhou, Y. Tong, R. Ren, K. Leung, E. Lau, J. Wong, X. Xing, N. Xiang, Y. Wu, C. Li, Z. Ma, , Y. Zhang, G. Shi, T. Lam, J. Wu, G. Goa, B. Cowling, B.J.and Yang, and Z. Leung, G. M.and Feng. Early transmission dynamics in wuhan, china, of novel coronavirus-infected pneumonia. N Engl J Med, 2020.

S. Marino, H. I. B. R. C. J., and D. E. Kirschner. A methodology for performing global uncertainty and sensitivity analysis in systems biology. Journal of theoretical biology, 254(1):178-196., 2008. doi: DOI:10.1016/j.jtbi.2008.04. 011.

C. N. Ngonghala, E. Iboi, S. Eikenberry, M. Scotch, C. R. MacIntyre, M. H. Bonds, and A. B. Gumel. Mathematical assessment of the impact of non-pharmaceutical interventions on curtailing the 2019 novel coronavirus. preprint, 2020 .

G. D. Nicholas, J. K. Adam, R. M. Eggo, A. Gimma, and W. J. Edmunds. Effects of non-pharmaceutical interventions on covid-19 cases, deaths, and demand for hospital services in the uk:a modelling study,. The Lancet Public Health, 2020 .

M. K. Njenga, J. Dawa, M. Nanyingi, J. Gachohi1, I. Ngere, M. Letko, C. F. Otieno, B. M. Gunn, and E. Osoro. Why is There Low Morbidity and Mortality of COVID-19 in Africa? The American Journal of Tropical Medicine and Hygiene, 103:564-569, 2020.

W. H. Organisation. Monitoring health for the sdgs, sustainable development goals. geneva: World health organization. World health statistics overview 2019, 2019.

K. Prem, Y. Liu, T. W. Russell, A. J. Kucharski, R. M. Eggo, N. Davies, C. for the Mathematical Modelling of Infectious Diseases COVID-19 Working Group, M. Jit, and P. Klepac. The effect of control strategies to reduce physical mixing on outcomes of the covid-19 epidemic in wuhan, china: a modelling study. Lancet Public Health, 2020 .

U. N. W. P. Prospects. World population. online https://population.un.org/wpp/, 7 2020. updated.

S. Ryu, H. Gao, J. Wong, E. Shiu, J. Xiao, M. Fong, , and B. Cowling. Nonpharmaceutical measures for pandemic influenza in non health care settings-international travel-related measures. Emerg Infect Dis, 26:961-966, 2020.

A. Siraj, A. Worku, K. Berhane, Y. Berhane, and D. Siraj. Early estimates of covid-19 infections in small, medium and large population clusters. $\underline{\text { s, }}$ 18, 2020.

R. Takele. Stochastic modelling for predicting covid-19 prevalence in east africa countries. Infectious disease modeling, 2020. 
P. van den Driessche and J. Watmough. Reproduction numbers and sub-threshold endemic equilibria for compartmental models of disease transmission. Mathematical Biosciences, 180, 2002a.

P. van den Driessche and J. Watmough. Reproduction numbers and sub-threshold endemic equilibria for compartmental models of disease transmission. Mathematical Biosciences, 180:29-48, 2002b.

N. van Doremalen, T. Bushmaker, D. H. Morris, M. G. Holbrook, A. Gamble, B. N. Williamson, A. Tamin, J. L. Harcourt, N. J. Thornburg, S. I. Gerber, J. O. Lloyd-Smith, E. de Wit, and V. J. Munster. Aerosol and surface stability of sars-cov-2 as compared with sars-cov-1. New England Journal Medicine, 382:1564-1567, 2020.

P. G. T. Walker, C. Whittaker, O. Wason, M. Baguelin, P. Winskill, H. A., B. Djafaara, Z. Cucunubá, and et.al. The global impact of covid-19 and strategies for mitigation and suppression. Science 369, 2020.

WHO. Novel coronavirus (2019-ncov) situation reports. online, 2020.

C. Worby and C. H-H. Face mask use in the general population and optimal resource allocation during the covid-19 pandemic. Nature Communication, 2020.

World-Bank. Measuring rural access: Using new technologies. washington dc. Transport and ICT., $18,2016$.

J. Wu, R. Dhingra, M. Gambhir, and J. Remais. Sensitivity analysis of infectious disease models: methods, advances and their application. Journal of The Royal Society Interface 10(86), 2013. doi: DOI:10.1098/rsif.2013.1018.

J. Xiao, E. Y. C. Shiu, H. Gao, J. Y. Wong, M. W. Fong, S. Ryu, , and B. J. Cowling. Nonpharmaceutical measures for pandemic influenza in non health care settings-personal protective and environmental measures. Emerg Infect Dis, 26:967-975, 2020.

\section{Appendix}

Theorem 2.1

\section{Proof. Positivity of $\Omega$}

To show $\Omega$ positive, first we show $S$ of the model is positive for all $t \geq 0$. To prove by contradiction: suppose, $S(t)=0, S^{\prime}(t)<0, E \geq 0, I_{s} \geq 0, I_{a} \geq 0, H_{c} \geq 0, I H_{m} \geq 0, R \geq 0, D \geq 0, E_{n v} \geq 0 t>0$. Then, using the first equation of system 11 we have,

$$
\frac{d S}{d t}(t)=\pi>0
$$

which contradicts $S^{\prime}(t)<0$. Thus, $\mathrm{S}(\mathrm{t})$ remains positive for $t \geq 0$. For the rest of population variables we can show positivity using Gronwall's inequality as follows.

$$
\frac{d E}{d t}=\gamma S-\sigma E-\mu E>-(\sigma+\mu) E
$$


Since $S(t)>0$ for $t \geq 0$ we can use Gronwalls inequality and $E \geq E_{0} e^{-(\sigma+\mu) t}>0$.

$$
\frac{d I_{a}}{d t}=(1-\theta) \sigma E-\rho I_{a}-\varepsilon I_{a}-y I_{a}-\mu I_{a}>-(\rho+\varepsilon+y+\mu) I_{a}
$$

since $E(t)>0$ for $t \geq 0$ we can use Gronwalls inequality and $I_{a} \geq I_{a 0} e^{-(\rho+\varepsilon+y+\mu) t} \geq 0$

$$
\frac{d I_{s}}{d t}=\theta \sigma E+\varepsilon I_{a}-e I_{s}-\omega I_{s}-\alpha_{1} I_{s}-\mu I_{s}>-\left(e+\omega+\alpha_{1}+\mu\right) I_{s}
$$

since $E(t)>0, I_{a}(t)>0$ for $t \geq 0$ we can use Gronwalls inequality and $I_{s} \geq I_{s 0} e^{-\left(e+\omega+\alpha_{1}+\mu\right) t} \geq 0$.

$$
\frac{d I H_{m}}{d t}=\rho I_{a}+e I_{s}-x I H_{m}-i I H_{m}-\mu I H_{m}>-(x+i+\mu) I H_{m} .
$$

Since $I_{a}(t)>0, I_{s}(t)>0$ for $t \geq 0$ we can use Gronwalls inequality and $I H_{m} \geq I H_{m 0} e^{-(x+i+\mu) t} \geq 0$.

$$
\frac{d H_{c}}{d t}=x I H_{m}-g H_{c}-\alpha_{2} H_{c}-\mu H_{c}>-\left(g+\alpha_{2}+\mu\right) H_{c}
$$

since $I_{s}(t)>0, I H_{m}(t)>0$ for $t \geq 0$ we can use Gronwalls inequality and $H_{c} \geq H_{c 0} e^{-\left(g+\alpha_{2}+\mu\right) t} \geq 0$

$$
\frac{d R}{d t}=g H_{c}+i I H_{m}+y I_{a}+\omega I_{s}-\mu R \geq-\mu R
$$

since $I_{a}(t)>0, I_{s}(t)>0, I H_{m}(t)>0, H_{c}(t)>0$ for $t \geq 0$ and $R(t) \geq R_{0} e^{-\mu t} \geq 0$

$$
\frac{d D}{d t}=\alpha_{1} I_{s}+\alpha_{2} H_{c}>0
$$

since $I_{s}(t)>0 H_{c}(t)>0$ for $t \geq 0$ and $D \geq D_{0} \geq 0 \dot{E}_{n v}=a I_{a}+b I_{s}-\varphi E_{n v} \leq(a+b) \bar{N}-\varphi E_{n v}$. Since $I_{a}(t)$ and $I_{s}(t)$ are less than the total population $N$ given as $\frac{\pi}{\mu}$ for all $t \geq 0$. Applying again the Gronwall inequality, for $0 \leq E_{n v}(0) \leq \frac{a+b}{\varphi} \frac{\pi}{\mu}$ leading to

$$
0 \leq E_{n v}(t) \leq \frac{a+b}{\varphi} \frac{\pi}{\mu}
$$

Hence all are non-negative for $t \geq 0$. Finally, the total number of population

$$
\begin{aligned}
N^{\prime}(t) & =S^{\prime}(t)+E^{\prime}(t)+I_{a}^{\prime}(t)+I_{s}^{\prime}(t)+I H_{m}^{\prime}(t)+H_{c}^{\prime}(t)+R^{\prime}(t)+D^{\prime}(t) \\
& =\pi-\mu N \\
N(t) & =\frac{\pi-Z e^{-\mu t}}{\mu} .
\end{aligned}
$$

Thus for initial data $0<N(0)<\frac{\pi}{\mu}$ we have $0 \leq N(t) \leq \frac{\pi}{\mu}$. Moreover, for the environment $E_{n v}$, we have $E_{n v}^{\prime}=$ $a I_{a}+b I_{s}-\varphi E_{n v} \leq(a+b) N-\varphi E_{n v}$ Since $I_{a}(t)$ and $I_{s}(t)$ are less than $N$ for all $t \geq 0$ applying Gronwalls inequality for $0 \leq E_{n v}(0) \leq \frac{(a+b)}{\varphi} \frac{\pi}{\mu}$ gives $0 \leq E_{n v}(t) \leq \frac{(a+b)}{\varphi} \frac{\pi}{\mu}$. 